TRANSACTIONS OF THE

AMERICAN MATHEMATICAL SOCIETY

Volume 364, Number 9, September 2012, Pages 4735-4761

S 0002-9947(2012)05504-8

Article electronically published on April 18, 2012

\title{
RANKS OF SELMER GROUPS IN AN ANALYTIC FAMILY
}

\author{
JOËL BELLAÏCHE
}

\begin{abstract}
We study the variation of the dimension of the Bloch-Kato Selmer group of a $p$-adic Galois representation of a number field that varies in a refined family. We show that, if we restrict ourselves to representations that are, at every place dividing $p$, crystalline, non-critically refined, and with a fixed number of non-negative Hodge-Tate weights, then the dimension of the Selmer group varies essentially lower-semi-continuously. This allows us to prove lower bounds for Selmer groups "by continuity", and in particular to deduce from a result of Bellaïche and Chenevier that the $p$-adic Selmer group of a modular eigenform of weight 2 of sign -1 has rank at least 1 .
\end{abstract}

\section{Contents}

1. Introduction 4735

2. Formalism of the variation of Selmer groups 4740

2.1. A general formalism 4740

2.2. An important special case 4742

2.3. Finiteness results 4744

2.4. Examples 4746

3. Trianguline families of $(\phi, \Gamma)$-modules 4748

3.1. Families of $(\phi, \Gamma)$-modules and their triangulations 4748

3.2. Triangulation of some refined families 4750

3.3. Finiteness results for the cohomology of $(\phi, \Gamma)$-modules 4753

3.4. The local Pottharst Selmer group $\quad 4755$

4. Proof of Theorem 104756

5. Application to modular forms 4758

Acknowledgment 4759

References $\quad 4760$

\section{INTRODUCTION}

We first introduce some notation and conventions that shall be used throughout this paper.

Let $K$ be a number field, $p$ an odd prime number, and $\Sigma$ a finite set of finite places of $K$ containing the set $\Sigma_{p}$ of places of $K$ above $p$. We denote by $G_{K, \Sigma}$ the Galois group of the maximal algebraic extension of $K$ unramified at all finite places that are not in $\Sigma$. For $v$ a place of $K$, we denote by $K_{v}$ the completion of $K$ at $v$, and by $G_{K_{v}}$, or $G_{v}$ when there is no ambiguity, the absolute Galois group of $K_{v}$. If $v \in \Sigma$, there is a natural morphism $i_{v}: G_{v} \rightarrow G_{K, \Sigma}$ well-defined up to conjugacy.

Received by the editors January 18, 2010 and, in revised form, October 17, 2010.

2010 Mathematics Subject Classification. Primary 11F80; Secondary 11F33.

(C) 2012 American Mathematical Society

Reverts to public domain 28 years from publication 
If $G_{K, \Sigma} \rightarrow \mathrm{GL}(V)$ is a representation, we will call by abuse its pre-composition with $i_{v}$ the restriction $V_{\mid G_{v}}$ of that representation $V$ to $G_{v}$. It is well-defined up to isomorphism.

All rings are commutative with unity. If $S$ is a ring, Spec $S$ is its spectrum in the sense of Grothendieck, and if $S$ is an affinoid algebra over $\mathbb{Q}_{p}, \operatorname{Sp} S$ is its attached rigid analytic space in the sense of Tate. If $x \in \operatorname{Sp} S$, we shall denote by $L(x)$ its field of definition, a finite extension of $\mathbb{Q}_{p}$.

The aim of this article is to study the variation of the Bloch-Kato Selmer group $H_{f}^{1}\left(G_{K, \Sigma}, V_{x}\right)$ (and its closely related variants $H_{g}^{1}\left(G_{K, \Sigma}, V_{x}\right)$ and $H_{e}^{1}\left(G_{K, \Sigma}, V_{x}\right)$ ) when the $p$-adic representation $V_{x}$ of $G_{K, \Sigma}$ varies along a family of representations.

To make this aim more precise, let us define a family of representations of a topological group $G$ of dimension $d$ over a topological ring $S$ as a locally free moduld 1 over $S$ of finite rank $d$, together with a continuous $S$-linear action of $G$. If $L$ is a topological field and $\chi: S \rightarrow L$ is a continuous and surjective morphism of algebras, $V_{\chi}:=V \otimes_{S, \chi} L$ is a continuous representation of $G_{K, \Sigma}$ over $L$ of dimension $d$. We shall say that the representation $V_{\chi}$ belongs to the family $V$. If $S$ is an affinoid algebra, $x \in \operatorname{Sp} S$ is a point of residue field $L(x)$ and $\chi_{x}: S \rightarrow L(x)$ is the corresponding character, we shall write $V_{x}$ instead of $V_{\chi_{x}}$.

The aim of this paper is to study how the dimensions of the spaces $H_{f}^{1}\left(G_{K, \Sigma}, V_{\chi}\right)$ change when $\chi$ runs along a suitable set of characters of $S$ (or of points in $\operatorname{Sp} S$ ), for suitable families $V$ of representations of $G_{K, \Sigma}$, in particular those carried by eigenvarieties.

That this variation is somewhat chaotic is already examplified by the case of the simplest and longest studied family of Galois representations: the Iwasawa family. Namely, take $K=\mathbb{Q}$ and $\Sigma=\Sigma_{p}$. Set $\Gamma=\mathbb{Z}_{p}^{*}$, and denote by $\Delta$ the subgroup of $(p-1)^{\text {th }}$-roots of unity in $\Gamma$, and by $\Gamma_{1}$ the kernel of the reduction modulo $p$ on $\Gamma$. The Teichmuller lift gives a canonical isomorphism $\Gamma=\Gamma_{1} \times \Delta$. Let $\eta_{i}$, for $i \in \mathbb{Z} /(p-1) \mathbb{Z}$ be the character $\Delta \rightarrow \mathbb{Z}_{p}^{*}$ that sends $t$ to $t^{i}$. The Iwasawa algebra is $\Lambda=\mathbb{Z}_{p}[[\Gamma]]$. We have $\Lambda=\prod_{i \in \mathbb{Z} /(p-1) \mathbb{Z}} \Lambda_{i}$ with $\Lambda_{i}=\Lambda \otimes_{\mathbb{Z}_{p}[\Delta], \eta_{i}} \mathbb{Z}_{p}=\mathbb{Z}_{p}\left[\left[\Gamma_{1}\right]\right] \simeq$ $\mathbb{Z}_{p}[[T]]$. We shall work in characteristic 0 , so set $\Lambda_{\mathbb{Q}_{p}}=\Lambda \otimes \mathbb{Q}_{p}$ and $\Lambda_{i, \mathbb{Q}_{p}}=\Lambda_{i} \otimes \mathbb{Q}_{p}$. If $L$ is any finite extension of $\mathbb{Q}_{p}$, a continuous character $\chi: \Gamma \rightarrow L^{*}$ defines a morphism of algebras $\Lambda_{\mathbb{Q}_{p}} \rightarrow L$ that factors through $\Lambda_{i, \mathbb{Q}_{p}}$ if and only if $\chi_{\mid \Delta}=\eta_{i}$. For $i \in \mathbb{Z} /(p-1) \mathbb{Z}$, the $i^{\text {th }}$ Iwasawa family is $V_{i}=\Lambda_{i, \mathbb{Q}_{p}}$, considered as a free $\Lambda_{i, \mathbb{Q}_{p}}$-module of rank one, with a (continuous) action of $G_{\mathbb{Q}, \Sigma_{p}}$ induced by the cyclotomic character $\omega_{p}: G_{\mathbb{Q}, \Sigma_{p}} \rightarrow \mathbb{Z}_{p}^{*}=\Gamma$. It is easily seen that the representation $\mathbb{Q}_{p}(n)$, that is, $\mathbb{Q}_{p}$ with the action of $G_{\mathbb{Q}, \Sigma_{p}}$ given by $\omega_{p}^{n}: G_{K, \Sigma} \rightarrow \mathbb{Q}_{p}^{*}, n \in \mathbb{Z}$, belongs to the family $V_{i}$ if and only if $n \equiv i(\bmod p-1)$. Assume that $i$ is odd. For $n \neq 1$ an odd integer it is well known, as a consequence of a result of Soulé, that $H_{f}^{1}\left(G_{K, \Sigma}, \mathbb{Q}_{p}(n)\right)=H_{e}^{1}\left(G_{K, \Sigma}, \mathbb{Q}_{p}(n)\right)=H_{g}^{1}\left(G_{K, \Sigma}, \mathbb{Q}_{p}(n)\right)$ is of dimension 1 if $n>1$ and is 0 if $n \leq-1$. Therefore, there is no generic value for $H_{f}^{1}$ (or $H_{g}^{1}$ or $\left.H_{e}^{1}\right)$ on the families $V_{i}$, that is, a value that is the dimension of $H_{f}^{1}\left(G_{K, \Sigma}, V_{\chi}\right)$ for all $V_{\chi}$ that belongs to $V_{i}$ and that are de Rham. For the sake of completeness, let us mention that $H_{f}^{1}\left(\mathbb{Q}_{p}(1)\right)=H_{e}^{1}\left(\mathbb{Q}_{p}(1)\right)=0$ while $\operatorname{dim} H_{g}^{1}\left(\mathbb{Q}_{p}(1)\right)=1$, and also that $\operatorname{dim} H_{\bullet}^{1}\left(V_{\chi}\right)=0$ (where $\bullet \in\{f, g, e\}$ ) when $V_{\chi}$ belongs to $V_{i}$ and is not de Rham. So the "most frequent" dimension of $H_{\bullet}^{1}$ is 0 , but there are infinitely

\footnotetext{
${ }^{1}$ We could define more generally a family to be a continuous pseudocharacter $G \rightarrow S$ of dimension $d$, as is done in $[\mathrm{BCh}$, but the generality provided will not be useful here.
} 
many exceptions (and moreover the set of exceptions is dense both in the Zariski topology and the rigid analytic topology), a pattern which is not very usual for a family over $\Lambda_{i, \mathbb{Q}_{p}}$, a Noetherian domain of dimension 1 .

Despite this chaotic behavior, it might be possible to prove the existence of a generic value for the dimension of the Selmer groups of the Galois representations in a family if we restrict our attention to the members of a family that are de Rham and that have a fixed number of negative Hodge-Tate weights. We shall indeed be able to prove a result of this type, but only under the additional assertion that the family is trianguline and that the representations concerned are non-critical. Let us review those two notions before stating our theorem:

Let $L$ be a finite extension of $\mathbb{Q}_{p}$. To a continuous representation of $G_{\mathbb{Q}_{p}}$ on a $L$-vector space $V$ of dimension $d$, one can attach (by results of Fontaine and ColmezCherbonnier) a $(\phi, \Gamma)$-module $D^{\dagger}(V)$ of rank $d$ (that is, a free $\mathcal{R}_{L}$-module $D$ of rank $d$ with a semi-linear action of $\phi$ and $\Gamma$, such that $\phi(D)$ spans $D$ over $\mathcal{R}_{L}$, where $\mathcal{R}_{L}$ is the Robba ring over $L$; see 3.1 .1 for a definition) which moreover is étale. Actually $D^{\dagger}$ is an equivalence of categories from the category of continuous finitedimensional representations of $G_{\mathbb{Q}_{p}}$ over $L$ to the category of étale $(\phi, \Gamma)$-modules, a full subcategory of the category of $(\phi, \Gamma)$-modules.

According to Colmez (Co1 $)$, a $(\phi, \Gamma)$-module $D$ over $\mathcal{R}_{L}$ is called trianguline if it is provided with a complete filtration $\mathrm{Fil}^{\bullet} D$ such that for $0 \leq i \leq d, \mathrm{Fil}^{i} D$ is a $(\phi, \Gamma)$-submodule of $D$, a direct summand as an $\mathcal{R}_{L}$-module, and of rank $i$. Such a filtration is called a triangulation, and it may not be unique if it exists; we say that $D$ is triangulable to express the fact that it has at least one triangulation, but that none is specified.

A triangulation on $D$ defines graded pieces $\operatorname{gr}^{i} D=\mathrm{Fil}^{i} D / \mathrm{Fil}^{i-1} D$ for $i=1, \ldots, d$ which are $(\phi, \Gamma)$-modules of rank one, and Colmez has shown that those objects are in natural bijection with continuous characters $\mathbb{Q}_{p}^{*} \rightarrow L^{*}$. A trianguline $(\phi, \Gamma)$ module thus defines a sequence of characters $\delta_{i}: \mathbb{Q}_{p}^{*} \rightarrow L^{*}, i=1, \ldots, d$, that we call its parameter. The weight $s_{i}$ of the character $\delta_{i}$ is defined as the negative of its derivative at 1 (caveat lector: this convention is the opposite of the one chosen by Colmez [Co1, §2.2], but it is the one of [BCh, $\$ 2.3 .3])$.

If $V$ is a representation of dimension $d$ over $L$ of $G_{\mathbb{Q}_{p}}$, we say that $V$ is triangulable if $D^{\dagger}(V)$ is, and that it is trianguline if we have chosen a triangulation of $D^{\dagger}(V)$. In general, if $X$ is an adjective describing a property of $(\phi, \Gamma)$-modules, we shall say that $V$ is $X$ if and only if $D^{\dagger}(V)$ is $X$.

It turns out (see [BCh, Prop 2.3.3]) that if $V$ is trianguline, the numbers $s_{i}$ attached to $D^{\dagger}(V)$ are, with multiplicity, the Hodge-Tate-Sen weights of $V$ (with the standard convention that the cyclotomic character $\omega_{p}$ has Hodge-Tate weight $-1)$. We shall say that a trianguline $(\phi, \Gamma)$-module is non-critical if all the $s_{i}$ are rational integers and if $s_{1}<s_{2}<\cdots<s_{d}$. A non-critical trianguline representation is always de Rham ([BCh, Prop 2.3.4]).

An important class of examples of trianguline representations are the crystalline representations $V$ such that the eigenvalues of the crystalline Frobenius $\phi$ on $D_{\text {crys }}(V)$ are in $L$ (the latter condition is of course always satisfied after replacing $L$ by a finite extension). More precisely, for such a representation $V$, any refinement of $V$, that is any full $\phi$-stable filtration of $D_{\text {crys }}(V)$, defines a triangulation, and conversely (see [BCh, Prop 2.4.1]). Assume for simplicity that $V$ has distinct Hodge-Tate weights. It is an easy consequence of the definitions that the 
refinement of $V$ is non-critical (in the sense of [BCh, §2.4.3]) if and only if $D^{\dagger}(V)$, with the attached triangulation, is non-critical (see [BCh, §2.4] for more details).

We shall say that a character $\delta: \mathbb{Q}_{p}^{*} \rightarrow L^{*}$ is exceptional if it is of the form $t \mapsto t^{n}$ or $t \mapsto t^{n}|t|$ for some integer $n \in \mathbb{Z}$. We shall say that a trianguline $(\phi, \Gamma)$-module $D$ is non-exceptional if all the characters $\delta_{i}$ of its parameter are not exceptional.

We have a similar notion of a trianguline family of representations $V$ of $G_{\mathbb{Q}_{p}}$ over an affinoid $S$, namely that the $(\phi, \Gamma)$-module over $\mathcal{R}_{S}$ (see $\$ 3.1 .1$ ) constructed by Berger and Colmez (see $\underline{\mathrm{BeCo}}$ and 93.1 .3$), D^{\dagger}(V)$, has a triangulation whose graded pieces are free $(\phi, \Gamma)$-modules of rank one of character type. Of course, if $V$ is such a family and $x \in \operatorname{Sp} S$ is a point of field of definition $L(x)$, then $V_{x}$ is a trianguline representation over $L(x)$. See $\$ 3.1 .5$ for more details.

We shall assume that $p$ is totally split in $K$. We define $H_{f}^{1}\left(G_{K, \Sigma}, V\right)$ as

$$
\operatorname{ker}\left(H^{1}\left(G_{K, \Sigma}, V\right) \rightarrow \prod_{v \in \Sigma_{p}} H^{1}\left(G_{v}, V \otimes B_{\text {crys }}\right)\right),
$$

and similarly for $H_{e}^{1}$ and $H_{g}^{1}$, with $B_{\text {crys }}$ replaced by $B_{\text {crys }}^{\phi=1}$ and $B_{\mathrm{dR}}$. Note that for simplicity we do not put conditions on places of $\Sigma-\Sigma_{p}$, but we could; see Remark 1 (i) after the theorem.

Theorem 1. Let $S$ be a an affinoid domain over $\mathbb{Q}_{p}$, and let $V$ be a locally free $S$-module of rank $d$ with a continuous $S$-linear action of $G_{K, \Sigma}$. Assume that the restriction of $V$ to $G_{K_{v}}$ is trianguline for every $v \in \Sigma_{p}$.

There exists an admissible covering of $S p S$ by open affinoid subdomains $S p S_{\alpha}$, such that upon replacing $S$ by $S_{\alpha}$, the following holds: for any sequence of integers $\underline{a}=\left(a_{v}\right)_{v \in \Sigma_{p}}$, with $0 \leq a_{v} \leq d$ for every $v$, let us call $Z_{\underline{a}}$ the set of points $x \in S p S$ such that the trianguline representation $\left(V_{x}\right)_{\mid G_{v}}$ is non-critical, non-exceptional, with exactly $a_{v}$ non-positive Hodge-Tate weights at every place $v$. Then there exists a natural integer $n_{\underline{a}}$, and a non-zero function $g \in S$, such that for every $x \in$ $Z_{\underline{a}}, \operatorname{dim} H_{f}^{1}\left(G_{K}, V_{x}\right) \geq n_{\underline{a}}$, and for every $x \in Z_{\underline{a}}$ such that $g(x) \neq 0$, one has $\operatorname{dim} H_{f}^{1}\left(G_{K}, V_{x}\right)=n_{\underline{a}}$.

Remark 1. (i) For $x \in Z_{\underline{a}}$, we have $\operatorname{dim} H_{g}^{1}\left(G_{K, \Sigma}, V_{x}\right)=\operatorname{dim} H_{f}^{1}\left(G_{K, \Sigma}, V_{x}\right)=$ $\operatorname{dim} H_{e}^{1}\left(G_{K, \Sigma}, V_{x}\right)$ (see Proposition 5). So the theorem is a theorem on the $H_{g}^{1}$ and $H_{e}^{1}$ as well.

Moreover, the theorem is also true for all the variants $H_{c}^{1}$ of $H_{f}^{1}, H_{g}^{1}$, $H_{e}^{1}$ defined as follows: choose an application $c: \Sigma \rightarrow\{0$, unr, $e, f, g, \emptyset\}$, such that $c(v) \in\{0$, unr, $\emptyset\}$ for $v \in \Sigma-\Sigma_{p}$, and define $H_{c}^{1}\left(G_{K, \Sigma}, V\right)$ as the set of $x \in H^{1}\left(G_{K, \Sigma}, v\right)$ such that, for all $v \in \Sigma$, then if $c(v)=0$ (resp. unr, $e, f, g, \emptyset)$, the natural image of $x$ in $H^{1}\left(G_{v}, V\right)$ (resp. $H^{1}\left(I_{v}, V\right)$, $\left.H^{1}\left(G_{v}, V \otimes B_{\text {crys }}^{\phi=1}\right), H^{1}\left(G_{v}, V \otimes B_{\text {crys }}\right), H^{1}\left(G_{v}, V \otimes B_{\mathrm{dR}}\right), 0\right)$ is 0 . The same proof works with trivial modifications.

(ii) The hypothesis that $p$ is totally split is technical and simplifies the statement. It is certainly easy to remove it, but we did not do the job. Also, the hypothesis of non-exceptionality could be weakened, and it would be possible to allow the sequence $\left(s_{i}\right)$, in the definition of non-critical, to be increasing instead of strictly increasing, but this would come at the cost of a more complicated statement (separating in particular the cases of $H_{f}^{1}, H_{e}^{1}$ and $H_{g}^{1}$ ). We have chosen to present the result in the simplest formulation that seems sufficient for most applications. 
(iii) It may happen that for a given $\underline{a}=\left(a_{v}\right)$, the set $Z_{\underline{a}}$ is finite, in which case the theorem is empty. But when $Z_{a}$ is infinite, the theorem states that there exists a "generic value" for $\operatorname{dim} H_{\bullet}^{\overline{1}}\left(G_{K, \Sigma}, V_{x}\right)$ for $x \in Z_{\underline{a}}$ (and $\bullet$ being indifferently $e, f$ or $g$ ), which is obviously well-determined, and that this value is also a lower bound.

(iv) It would be interesting to have some control on the set of $x \in Z_{\underline{a}}$ such that $\operatorname{dim} H_{\bullet}^{1}\left(G_{K, \Sigma}, V_{x}\right)>n_{\underline{a}}$ (besides the fact that it is not Zariski-dense). The information given by the proof does not seem useable.

Let us just say a few words about the method of the proof. The Selmer groups are defined as the kernel of a natural map from a global Galois cohomology group to a product of a local Galois cohomology group. For example $H_{f}^{1}\left(G_{K, \Sigma}, V\right)=$ $\operatorname{ker}\left(H^{1}\left(G_{K, \Sigma}, V\right) \rightarrow \prod_{v \in \Sigma_{p}} H^{1}\left(G_{v}, V \otimes B_{\text {crys }}\right)\right)$. The same definition makes sense when $V$ is a family of representations over an algebra $S$ instead of an individual one. A formal argument, developed in $\$ 2.1$, shows that when the involved cohomology modules and also the $H^{2}\left(G_{K, \Sigma}, V\right)$ are finite over $S$, the resulting Selmer groups behave well in families. Unfortunately, the modules $H^{1}\left(G_{v}, V \otimes B_{\text {crys }}\right)$ are not finite in general, which explains the chaotic behavior of the $H_{f}^{1}$ described at the beginning of this article. To circumvent this problem, we use an idea due to Jay Pottharst, himself generalizing both an idea of Greenberg (in the ordinary case) and an idea of Bellaïche-Chenevier ( $\mathrm{BCh}$, Chapter 2] in the non-necessary ordinary case but only for certain types of representations) to replace the $H_{f}^{1}$ by another type of Selmer group, defined using $(\phi, \Gamma)$-modules and their cohomology, that we call the Pottharst Selmer group $H_{\text {pot }}^{1}$. We prove that the cohomology groups involved in its definition are of finite type in a family (cf. $\S \S 2.3$ and 3.3 ), and that for non-critical representations, the $H_{\text {pot }}^{1}$ and $H_{f}^{1}$ are essentially the same (an easy consequence of the results of $[\mathrm{BCh}$, chapter 2], and also a variant of a theorem of $[\mathrm{P}]$ ); see Proposition 5. This, together with our formal work of \$2.1, gives the theorem.

Of course, our theorem is interesting only to the extent that we are able to check that natural families are trianguline. By natural families we mean essentially the families carried by the eigenvarieties, and more generally the refined families defined in [BCh, Chapter IV]: they are families of representations over an affinoid algebra $S$ with a dense set of classical points $Z \subset \operatorname{Sp} S$ where the representations $\rho_{z}$ are crystalline and provided with a refinement such that for every $i=1, \ldots, d$, the $i^{\text {th }}$ eigenvalue of the crystalline Frobenius (for the order given by the refinement) renormalized by the $i$ th Hodge-Tate weight (in increasing order) $\kappa_{i}(z)$, extends to an analytic function defined on all $X$ (namely $p^{-\kappa_{i}(z)} F_{i}(z)$ extends to an analytic function over $X)$. There are several other minor requirements in this definition, which are recalled below in $\$ 3.2 .1$

Our second main theorem (Theorem 2) states that, if $V$ is such a family $S$ which has rank 2, and $x \in \operatorname{Sp} S$ is a classical point such that $V_{x}$ is non-critical, there exists an affinoid neighborhood of $x$ on which $V$ becomes trianguline. The hypothesis of non-criticality is needed, as shown by [BCh, Example 2.5.8]. We conjecture (cf. Conjecture 1) that the same result holds in any rank. Note that the formal counterpart of this statement, that is, the same statement where "an affinoid neighborhood of $x$ " is replaced by the "completion of the local ring of $S$ at $x "$, is known ([BCh, Theorem 2.5.6]). It seems that Ruochuan Liu has made some progress toward this conjecture ([Liu4], Liu5]). 
As an application, we can extend a theorem of [BCh] which states that for a modular eigenform $k$ of weight $k=2 k^{\prime} \geq 4$ and of sign -1 , the Selmer group $H_{f}^{1}\left(\mathbb{Q}, V_{f}\left(k^{\prime}\right)\right)$ has dimension at least 1 (where $V_{f}$ is the $p$-adic Galois representations attached to $f$ ) to the case of forms of weight 2 , and in particular to elliptic curves 2

This result, a consequence of the Bloch-Kato conjecture and of the Birch and Swinnerton-Dyer conjecture, was previously known only for primes $p$ that are potentially ordinary for $f$ : the ordinary case has two completely different proofs, one of Nekovar ([N1], [N2]) and one of Skinner and Urban ([SkU]), and the potentially ordinary case is in $\mathrm{N3}$.

\section{Formalism of the variation of Selmer groups}

2.1. A general formalism. Let $R$ be a commutative domain and let $\mathcal{A}$ and $\mathcal{A}^{\prime}$ be two abelian $R$-linear categories. We let $\left(H_{\mathcal{A}}^{i}, \delta\right)$ be a cohomological functor from $\mathcal{A}$ to the category of $R$-modules, and we let $\left(H_{\mathcal{A}^{\prime}}^{i}, \delta\right)$ be another cohomological functor from $\mathcal{A}^{\prime}$ to the category of $R$-modules. When no confusion can result, we shall drop the $\mathcal{A}$ or $\mathcal{A}^{\prime}$ from the notation. We say that an object $A$ of $\mathcal{A}$ is torsion-free if for every $0 \neq f \in R$, the endomorphism $f$ of $A$ is a monomorphism. We write $A / f A$ for its cokernel. Note that $f$ acts as 0 on $A / f A$, so by functoriality it does on $H^{1}(A / f A)$ as well, which is therefore naturally an $R / f$-module. Similar definitions and considerations apply for $\mathcal{A}^{\prime}$.

Finally, we suppose given two torsion-free objects $V \in \mathcal{A}, V^{\prime} \in \mathcal{A}^{\prime}$, a morphism of $R$-modules $u: H^{1}(V) \rightarrow H^{1}\left(V^{\prime}\right)$, and for every $0 \neq f \in V$, a morphism of $R / f$-modules $u_{f}: H^{1}(V / f V) \rightarrow H^{1}\left(V^{\prime} / f V^{\prime}\right)$ such that the following diagram commutes:

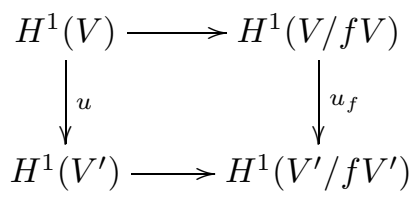

In this context, we define the Selmer modules $S(V)$ as ker $u$ and $S(V / f V)$ as $\operatorname{ker} u_{f}$.

From now on, we assume that $R$ is Noetherian and call $\operatorname{Pr}(R)$ the set of principal prime ideals in $R$. If $M$ is an $R$-module, we call $\mathrm{rk}_{R} M$ its generic rank, that is, the dimension over $\operatorname{Frac}(R)$ of $M \otimes \operatorname{Frac}(R)$, which is a natural integer or $+\infty$.

Proposition 1. Let $(f) \in \operatorname{Pr}(R)$. Assume that $H^{0}\left(V^{\prime} / f V^{\prime}\right)=0$. Then the natural $R / f$-linear map $S(V) \otimes_{R} R / f \rightarrow S(V / f V)$ is injective, and its cokernel has generic rank no more that $\mathrm{rk}_{R / f} H^{2}(V)[f]+\operatorname{rk}_{R / f}\left(H^{1}\left(V^{\prime}\right) / u\left(H^{1}(V)\right)\right)[f]$. Moreover, if $S(V) \otimes_{R} R / f, H^{2}(V)[f]$ and $\left(H^{1}\left(V^{\prime}\right) / u\left(H^{1}(V)\right)\right)[f]$ are all finite over $R / f$, then so is $S(V / f V)$.

\footnotetext{
${ }^{2}$ In the case of elliptic curves, our result is also a consequence of two recent results proving the parity conjecture for elliptic curves: one of Kim ( $\mathrm{Kim}]$ ) and one of T. and V. Dokchitser D]. Those results use completely different methods - from each other and from ours. We thank Emerton and Nekovar for pointing out those references.
} 
Proof. We have the following commutative diagram where rows and columns are exact sequences:

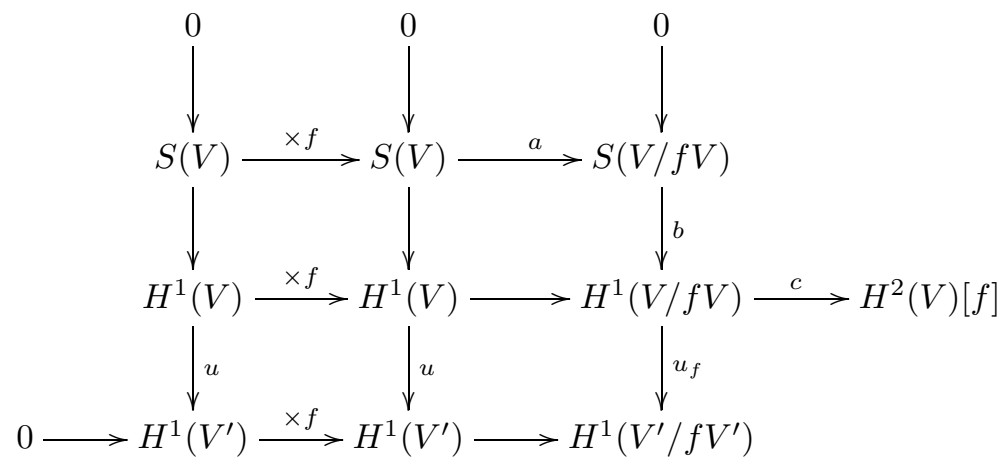

Indeed, the commutativity of the diagram follows immediately from the commutativity of (1), the $R$-linearity of $u$, and the definitions of $S(V)$ and $S(V / f V)$. The exactness of the second and third lines comes from the long exact sequences of cohomology attached to the short exact sequence $0 \rightarrow V \rightarrow V \rightarrow V / f V \rightarrow 0$, and similarly for $V^{\prime}$ (those exact sequences being exact by our hypothesis that $V$ and $V^{\prime}$ are torsion-free). The only fact that may need a justification is the injectivity of the map labeled $\times f$ on the third line, but this comes from our hypothesis that $H^{0}\left(V^{\prime} / f V^{\prime}\right)=0$. The morphisms on the first line are defined by restriction of the morphisms of the second line, which makes the first line obviously a complex. An easy diagram-chasing argument (a part of the Snake Lemma, in fact) shows that it is exact.

This diagram already shows that the map $S(V) \otimes_{R} R / f \rightarrow S\left(V_{f}\right)$ is injective.

From the diagram above, we deduce another one:

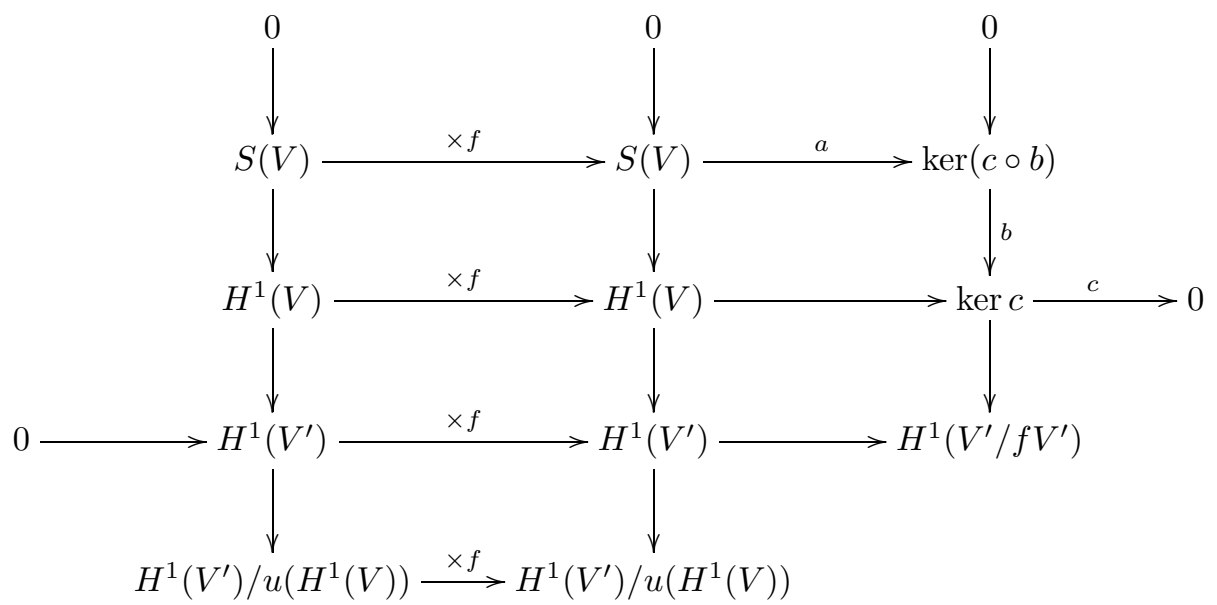

From the Snake Lemma, we deduce that the cokernel of the map a (that is, $\operatorname{ker}(c \circ b) / a(S(V)))$ is isomorphic to $\left(H^{1}\left(V^{\prime}\right) / u\left(H^{1}(V)\right)\right)[f]$. But $S(V / f V) / \operatorname{ker}(c \circ b)$ is isomorphic to a submodule of $H^{2}(V)[f]$. The proposition follows.

Remark 2. Actually the hypotheses that $R$ is a domain and that $(f)$ is prime are only used for the convenience of stating the result in terms of generic ranks. Without these hypotheses, we still get that if $H^{0}\left(V^{\prime} / f V^{\prime}\right)=0$ and $f$ is a monomorphism 
of $V$ and $V^{\prime}$, the map $S(V) / f S(V) \rightarrow S(V / f V)$ is injective, and it is surjective whenever $H^{2}(V)[f]$ and $\left(H^{1}\left(V^{\prime}\right) / u\left(H^{1}(V)\right)\right)[f]$ are 0 .

Corollary 1. Assume that $R$ is a domain and that $S(V), H^{2}(V)$ and $H^{1}\left(V^{\prime}\right)$ are finite as $R$-modules. Let $r$ be the generic rank of $S(V)$. Then for all $(f) \in \operatorname{Pr}(R)$ such that $H^{0}\left(V^{\prime} / f V^{\prime}\right)=0, S(V / f V)$ is finite over $R / f$, we have $\operatorname{rk}_{R / f} S(V / f V) \geq$ $r$, and for almost all such $(f)$, we have $\operatorname{rk}_{R / f} S(V / f V)=r$.

Proof. By the proposition, the map $S(V) \otimes_{R} R / f \rightarrow S(V / f V)$ is injective for all $f$ such that $H^{0}\left(V^{\prime} / f V^{\prime}\right)=0$, and by Nakayama's Lemma, $\operatorname{rk}_{R / f}\left(S(V) \otimes_{R} R / f\right) \geq$ $\operatorname{rk}_{R} S(V)=r$ for all $(f)$. Hence the first inequality. Also the fact that $S(V / f V)$ is of finite type follows from the proposition, since $S(V) \otimes R / f$ is finite over $R / f$ because $S(V)$ is finite over $R$, and $H^{2}(V)[f]$ and $H^{1}\left(V^{\prime}\right) / u\left(H^{1}(V)\right)[f]$ are finite over $R / f$ since they are finite over $R$ as submodules of the finite modules $H^{2}(V)$ and $H^{1}\left(V^{\prime}\right) / u\left(H^{1}(V)\right)$ over the Noetherian ring $R$.

Since $S(V)$ is a finite $S$-module, it is flat on an open subset of $\operatorname{Spec} R$ whose complement is a finite set of points. We thus have $\operatorname{rk}_{R / f} S(V) \otimes_{R} R / f=r$ for all maximal ideals $(f)$ that are not in that finite set of points. Moreover, the finite $S$-modules $H^{2}(V)$ and $H^{1}\left(V^{\prime}\right) / u\left(H^{1}(V)\right)$ have no $f$-torsion for almost all $(f)$, so by the proposition, $\operatorname{rk}_{R / f} S(V) \otimes_{R} R / f=\operatorname{rk}_{R / f} S(V / f V)$ for almost all $(f) \in \operatorname{Pr}(R)$. The corollary follows.

2.2. An important special case. In the above formalism, the morphisms $u$ and $u_{f}$ are $a d$ hoc. Often, however, they appear naturally as follows.

Again $R$ is a commutative domain, and we now suppose it to be Noetherian, and $\mathcal{A}$ and $\mathcal{A}^{\prime}$ are two abelian $R$-linear categories with two cohomological functors toward the category of $R$-modules both denoted $\left(H^{i}\right)$ (or $\left(H_{\mathcal{A}}^{i}\right)$ and $\left(H_{\mathcal{A}^{\prime}}^{i}\right)$ when we really need to distinguish them). We assume given in addition an $R$-linear functor $F$ from $\mathcal{A}$ to $\mathcal{A}^{\prime}$ and a morphism of functors on $\mathcal{A}, a: H_{\mathcal{A}}^{1} \rightarrow H_{\mathcal{A}^{\prime}}^{1} \circ F$.

Then, given an object $V$ of $\mathcal{A}$, an object $V^{\prime}$ of $\mathcal{A}^{\prime}$ and a morphism $b: F(V) \rightarrow V^{\prime}$ in $\mathcal{A}^{\prime}$, we can retrieve the situation of the preceding subsection as follows. We define $u: H_{\mathcal{A}}^{1}(V) \rightarrow H_{\mathcal{A}^{\prime}}^{1}\left(V^{\prime}\right)$ as the composition $H_{\mathcal{A}}^{1}(V) \stackrel{a}{\rightarrow} H_{\mathcal{A}^{\prime}}^{1}(F(V)) \stackrel{H^{1}(b)}{\rightarrow} H_{\mathcal{A}^{\prime}}^{1}\left(V^{\prime}\right)$, and $S(V)=S\left(V, V^{\prime}, b\right)$ as $\operatorname{ker} u$.

Now if $I$ is any ideal of $R$, we can define a subobject $I V$ of $V$ as the sum in $V$ of $f V$ for $f \in I$, and therefore also a quotient object $V / I V$. Similar definitions apply to objects in $\mathcal{A}^{\prime}$. We shall make the following assertion on the functor $F$ : for every ideal $I$ of $R$ and every object $V$ of $\mathcal{A}$, there is an isomorphism $F(V / I V)=F(V) / I F(V)$, functorial in $V$. We say that $F$ commutes with quotients.

The map $b: F(V) \rightarrow V^{\prime}$ induces a map $b_{I}: F(V / I V) \rightarrow V^{\prime} / I V^{\prime}$. By the construction of the preceding paragraph where $R$ is replaced by $R / I, V$ by $V / I V$, $V^{\prime}$ by $V^{\prime} / I V^{\prime}$ and $b$ by $b_{I}$, we get a map $u_{I}: H^{1}(V / I V) \rightarrow H^{1}\left(V^{\prime} / I V^{\prime}\right)$ and a $R / I$-module $S(V / I V)=\operatorname{ker}\left(u_{I}\right)$. If $J \subset I$ are ideals of $R$, we have by construction a commutative diagram:

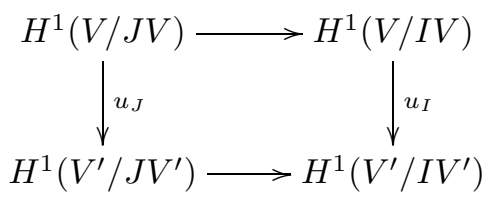


We shall say that a sequence $f_{1}, \ldots, f_{n}$ of elements in $R$ is $V$-regular if $f_{1}$ is a monomorphism of $V, f_{2}$ is a monomorphism of $V / f_{1} V, f_{3}$ is a monomorphism of $V /\left(f_{1}, f_{2}\right) V$, etc. Note that when $\mathcal{A}$ is the category of $R$-modules, this agrees with the standard definition (cf. e.g. [E]) of a regular sequence for a module $V$. In general, we shall say that an object $V \in \mathcal{A}$ is quasi-free if every $R$-regular sequence is a $V$-regular sequence. Similar definitions apply for $\mathcal{A}^{\prime}$.

Proposition 2. Assume that $V$ and $V^{\prime}$ are quasi-free. Assume that for all ideals $I$ of $A, H_{\mathcal{A}}^{i}(V / I V)$ and $H_{\mathcal{A}^{\prime}}^{i}\left(V^{\prime} / I V^{\prime}\right)$ are finite over $R / I$ for all $i \geq 0$ and that they are 0 for $i>d$ for some fixed integer $d$.

Let $r$ be the generic rank of $S(V)$. Then for all prime $R$-regular ideals $\mathfrak{p}$ of $R$ satisfying $H^{0}\left(V^{\prime} / \mathfrak{p} V^{\prime}\right)=0$, we have $\operatorname{rk}_{R / \mathfrak{p}} S(V / \mathfrak{p} V) \geq r$, and there exists a fixed non-zero element $g \in R$ such that equality holds if $\mathfrak{p}$ does not contain $g$.

Proof. Let $\mathfrak{p}$ be a prime $R$-regular ideal, and let $\mathfrak{p}=\left(f_{1}, \ldots, f_{n}\right)$ where $\left(f_{1}, \ldots, f_{n}\right)$ is an $R$-regular sequence. Let us set $I_{k}=\left(f_{1}, \ldots, f_{k}\right)$, so $I_{n}=\mathfrak{p}$. We first prove that if $H^{0}\left(V^{\prime} / \mathfrak{p} V^{\prime}\right)=0$, then for $0 \leq k \leq n, H^{0}\left(V^{\prime} / I_{k} V^{\prime}\right) \otimes_{R} R_{\mathfrak{p}}=0$. Indeed, this is true for $k=n$. Assume that it is true for some $k$; we shall show that it is true for $k-1$. We have, since $V^{\prime}$ is quasi-free, an exact sequence

$$
0 \rightarrow V^{\prime} / I_{k-1} V^{\prime} \stackrel{f_{k}}{\longrightarrow} V^{\prime} / I_{k-1} V^{\prime} \rightarrow V^{\prime} / I_{k} V^{\prime} \rightarrow 0
$$

Taking the long exact sequence of cohomology, we get in particular

$$
H^{0}\left(V^{\prime} / I_{k-1} V^{\prime}\right) / f_{k} \hookrightarrow H^{0}\left(V^{\prime} / I_{k-1} V^{\prime}\right) .
$$

Tensorizing by $R_{\mathfrak{p}}$, which is flat, and using the induction hypothesis, we get that $\left(H^{0}\left(V^{\prime} / I_{k-1} V^{\prime}\right) / f_{k}\right) \otimes R_{\mathfrak{p}}=0$. Since $f_{k} \in \mathfrak{p}$, we conclude by Nakayama's Lemma.

We now prove by induction on $k$ that for $\mathfrak{p}$ as above, and $0 \leq k \leq n$, we have

$$
\left(S(V) / I_{k} S(V)\right) \otimes R_{\mathfrak{p}} \hookrightarrow S\left(V / I_{k} V\right) \otimes R_{\mathfrak{p}}
$$

For $k=0$ there is nothing to prove. To simplify notation, let us denote $V / I_{k-1} V$ by $\bar{V}$ and $V^{\prime} / I_{k-1} V^{\prime}$ by $\bar{V}^{\prime}$, and let us set $f=f_{k}$. Since $V$ and $V^{\prime}$ are quasi-free, $f$ is a monomorphism of $\bar{V}$ and $\bar{V}^{\prime}$. We only have to prove that $S(\bar{V} / f \bar{V}) \hookrightarrow S(\bar{V}) / f S(\bar{V})$. Similar to the proof of Proposition 1, we get a diagram

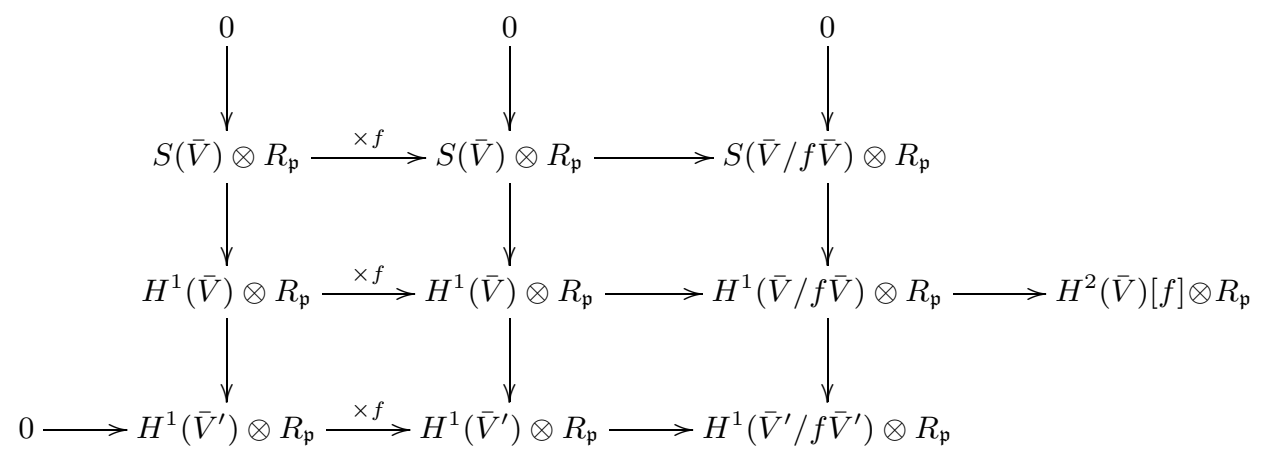

using long exact sequences of cohomology tensorized by the flat $R$-module $R_{\mathfrak{p}}$. The commutativity of the diagram is obvious except for the commutativity of the lower right square, which is (2), and the exactness of the second line is clear, while the exactness of the third line uses the fact that $H^{0}\left(\bar{V}^{\prime} / f \bar{V}^{\prime}\right) \otimes R_{\mathfrak{p}}=H^{0}\left(V^{\prime} / I_{k} V^{\prime}\right) \otimes$ $R_{\mathfrak{p}}=0$ that we have just proved. The exactness of the first line follows (by a part 
of the Snake Lemma), so we get $(S(\bar{V}) / f S(\bar{V})) \otimes R_{\mathfrak{p}} \hookrightarrow S(\bar{V} / f \bar{V}) \otimes R_{\mathfrak{p}}$, which completes our induction step.

Taking $k=n$, we get $(S(V) / \mathfrak{p} S(V)) \otimes R_{\mathfrak{p}} \hookrightarrow S(V / \mathfrak{p} V) \otimes R_{\mathfrak{p}}$, but since $S(V) / \mathfrak{p} S(V)$ and $S(V / \mathfrak{p} V)$ are already $R / \mathfrak{p}$-modules, we simply get $S(V) / \mathfrak{p} S(V) \hookrightarrow S(V / \mathfrak{p} V)$, so by Nakayama's Lemma, $\operatorname{rk}_{R / \mathfrak{p}} S(V / \mathfrak{p} V) \geq \operatorname{rk}_{R} S(V)$.

We now prove that this inequality is often an equality. There exists a non-empty open set of Spec $R$ over which $H^{i}(V), H^{i}\left(V^{\prime}\right)$ and $H^{i}\left(V^{\prime}\right) / u\left(H^{i}(V)\right)$ are flat for all $0 \leq i \leq d$, since those modules are finite. Take $g$ as any non-zero element in an ideal of definition of the closed set that is the complement of that open set. We thus know that $H^{i}(V) \otimes_{R} R[1 / g], H^{i}\left(V^{\prime}\right) \otimes_{R} R[1 / g]$ and $\left(H^{i}\left(V^{\prime}\right) / u\left(H^{i}(V)\right)\right) \otimes_{R} R[1 / g]$ are flat over $R[1 / g]$ for all $0 \leq i \leq d$ and 0 for $i>d$.

Let $\mathfrak{p}=\left(f_{1}, \ldots, f_{n}\right)$ be a prime ideal, with $f_{1}, \ldots, f_{n}$ a regular sequence and such that $H^{0}(V / \mathfrak{p} V)=0$ as above, and also assume that $g \notin \mathfrak{p}$. We shall prove by induction on $k$ the following statement: the natural map $\left(S(V) / I_{k} S(V)\right) \otimes R_{\mathfrak{p}} \rightarrow$ $S\left(V / I_{k} V\right) \otimes R_{\mathfrak{p}}$ is an isomorphism, the modules $H^{i}\left(V / I_{k} V\right) \otimes R_{\mathfrak{p}}$ and $H^{i}\left(V^{\prime} / I_{k} V^{\prime}\right) \otimes$ $R_{\mathfrak{p}}$ are flat over $R_{\mathfrak{p}} / I_{k} R_{\mathfrak{p}}$ for all $0 \leq i \leq d$ and 0 for $i>d$, and the module $\left(H^{1}\left(V^{\prime} / I_{k} V^{\prime}\right) / u_{I_{k}}\left(H^{1}\left(V / I_{k} V\right)\right)\right) \otimes R_{\mathfrak{p}}$ is flat over $R_{\mathfrak{p}} / I_{k} R_{\mathfrak{p}}$.

If $k=0$, this results from the fact that $R_{\mathfrak{p}}$ is an $R[1 / g]$-module and the universal nature of flatness.

Assume the assertion is true for $k-1$, and set as above $\bar{V}=V / I_{k} V$ (and similarly for $\left.V^{\prime}\right)$ and $f=f_{k}$. So we know that $H^{i}(\bar{V})$ and $H^{i}\left(\bar{V}^{\prime}\right)$ are flat over $R_{\mathfrak{p}} / I_{k-1} R_{\mathfrak{p}}$ for all $0 \leq i \leq d$ and 0 for $i>d$, and that $H^{1}\left(\bar{V}^{\prime}\right) / u_{I_{k-1}}\left(H^{1}(\bar{V})\right)$ is also flat over $R_{\mathfrak{p}} / I_{k-1} R_{\mathfrak{p}}$. We prove that the same assertions hold with $\bar{V}$ replaced by $\bar{V} / f \bar{V}$, by descending induction on $i$ (the result being true for $i>d$ by hypothesis). We have exact sequences

$$
0 \rightarrow\left(H^{i}(\bar{V}) / f H^{i}(\bar{V})\right) \otimes R_{\mathfrak{p}} \rightarrow\left(H^{i}(\bar{V} / f \bar{V})\right) \otimes R_{\mathfrak{p}} \rightarrow\left(H^{i+1}(\bar{V})[f]\right) \otimes R_{\mathfrak{p}},
$$

and similarly for $V^{\prime}$. From the fact that $H^{i+1}(\bar{V}) \otimes R_{\mathfrak{p}}$ is flat over $R_{\mathfrak{p}} / I_{k-1} R_{\mathfrak{p}}$, in particular has no $f$-torsion, we deduce that the second map is an isomorphism, so $H^{i}(\bar{V} / f \bar{V}) \otimes R_{\mathfrak{p}}$ is flat over $R_{\mathfrak{p}} / I_{k} R_{\mathfrak{p}}$. The same holds for $V^{\prime}$. By right exactness of the tensor product, the flatness of the module

$$
\left(H^{1}\left(V^{\prime} / I_{k} V^{\prime}\right) / u_{I_{k}}\left(H^{1}\left(V / I_{k} V\right)\right)\right) \otimes R_{\mathfrak{p}}
$$

then follows from the induction hypothesis.

Finally, we can apply the proof of Proposition 1 (and Remark 2) to see that $S(\bar{V} / f \bar{V}) \otimes R_{\mathfrak{p}}=(S(\bar{V}) / f S(\bar{V})) \otimes R_{\mathfrak{p}}$. The induction step, and therefore the proposition, follows.

Corollary 2. Same hypothesis and notation as the proposition above. There exists $a g \in R, g \neq 0$, such that for all regular closed points $x$ of Spec $R$ such that $H^{0}\left(V_{x}^{\prime}\right)=0$, we have $\operatorname{dim} S\left(V_{x}\right) \geq r$ with equality when $g(x) \neq 0$.

Proof. A closed point $x$ corresponds to a maximal ideal $\mathfrak{p}$, and we have $V_{x}=V / \mathfrak{p} V$. If $x$ is regular, the ring $R_{\mathfrak{p}}$ is a regular local ring, therefore the ideal $\mathfrak{p}$ is $R$-regular by [E, Cor 10.15].

2.3. Finiteness results. The following proposition generalizes to any $p$-complete algebra $S$ a similar result of Greenberg for $S$ a local ring with finite residue field complete for the topology defined by its maximal ideal ([G, Remark 3.5.1]) and an earlier result of Tate (for $S=\mathbb{Z}_{p}$; see $[\mathrm{T}, \S 2]$ and [Ru, Prop B.2.7]). A part of its proof has been given to us by Brian Conrad. 
Proposition 3. Let $G=G_{K, \Sigma}$ or $G=G_{v}$ for $v$ some place of $K$, or let $G=I_{v}$ (the inertia subgroup of $G_{v}$ for $v$ a place of $K$ not dividing $p$ ). Let $S$ be a $p$ complete commutative ring, and let $M$ be a finite free $S$-module with a continuous (for the p-adic topology) action of $G$. For any integer $i \geq 0$, let $H^{i}(G, M)$ denote the continuous cohomology group of $G$ with coefficients $M$. Then $H^{i}(G, M)$ is finite over $S$ and is 0 if $i>2$. Moreover, $H^{i}(G, M) \otimes_{\mathbb{Z}_{p}} \mathbb{Q}_{p}=H^{i}\left(G, M \otimes_{\mathbb{Z}_{p}} \mathbb{Q}_{p}\right)$.

Proof. We claim that for every finite $S$-submodule $Y$ of $H^{i}(G, M), H^{i}(G, M) / Y$ has no non-zero p-divisible element. Let $Z$ be a submodule of finite type of $Z^{i}(G, M)$ (closed continuous cochains) that maps surjectively onto $Y$ (take for $Z$ the submodule generated by arbitrary lifts of the elements of a finite generating family of $Y)$. Since $Z$ is finite, it is closed $p$-adically in $Z^{i}(G, M)$. From this assume that one has a family $\left(x_{n}\right)_{n \in \mathbb{N}}$ of elements in $H^{1}(G, M) / Y$ with $p x_{n+1}=x_{n}$. Choose representatives $f_{n}$ of $x_{n}$ in $Z^{i}(G, M)$. We can write $p f_{n+1}=f_{n}+z_{n}+\partial h_{n}$ with $z_{i} \in Z$ and $h_{n} \in C^{i-1}(G, M)$. From this it follows that $f_{0}=\sum_{n \geq 0} p^{n} z_{n}+\partial\left(\sum_{n \geq 0} p^{n} h_{n}\right) \in$ $Z+B^{i}(G, M)$, which shows that $x_{0}=0 \in H^{i}(G, M) / Y$.

Exactly as in Tate [T, Corollary to Prop. 2.1], we can now prove the implication if $H^{i}(G, M) / p$ is finite over $S / p$, then $H^{i}(G, M)$ is finite over $S$. Indeed, lifting each element of a finite generating family of $H^{i}(G, M) / p$ gives a finite family in $H^{1}(G, M)$ that generates an $S$-submodule $Y$ such that $H^{i}(G, M)=p H^{i}(G, M)+Y$. This implies that $H^{i}(G, M) / Y$ is $p$-divisible, hence 0 by the claim already proved. So $H^{i}(G, M)=Y$ is finitely generated.

Now we prove that $H^{i}(G, M) / p$ is finite over $S / p$. Since this module is a submodule of $H^{1}(G, M / p)$, we are reduced to prove that the latter is finite over $S / p$. But $M / p$ is a finite free module over $S / p$. Remember that $G$ acts on it continuously for its quotient topology, that is, the discrete topology. Let $e_{i}$ be a basis of $M / p$, and let $U_{i}$ be a normal open subgroup of $G$ that fixes $e_{i}$. Then $U=\bigcap U_{i}$ is an open normal subgroup of $G$ that acts trivially on $M / p$. By the Hochschild-Serre spectral sequence, since $G / U$ is finite, we are reduced to proving that the modules $H^{i}(U, M / p)$ are finite over $S / p$. But since $U$ acts trivially, we have $H^{i}(U, M / p)=$ $H^{i}\left(U, \mathbb{F}_{p}\right) \otimes_{\mathbb{F}_{p}} M / p$, and since $H^{i}\left(U, \mathbb{F}_{p}\right)=\operatorname{Hom}_{\text {cont }}\left(U, \mathbb{F}_{p}\right)$ is well known to be finite (see [Ru, Prop B.2.7]), it follows that $H^{i}(U, M / p)$ is finite over $S / p$.

So we have proved that $H^{i}(G, M)$ is finite over $S$. The fact that $H^{i}(G, M)=0$ if $i>2$ results, in view of the above proof, from the well-known fact that $H^{1}\left(U, \mathbb{F}_{p}\right)=$ 0 for $i>2$, where $U$ is as above (remember our running assumption that $p>2$ ).

The "moreover" is then proved exactly as in [T, Prop. 2.3].

Corollary 3. Let $S$ be a reduced affinoid algebra, and let $V$ be a locally-free finite type module over $S$ with a continuous action of $G$ (the same notation as in the proposition). Then $H^{i}(G, V)$ is a finite type $S$-module and is 0 if $i>2$.

Proof. According to Chenevier's lemma (cf. Ch, Lemma 3.18]), there is an admissible covering of $\operatorname{Sp} S$ by affinoid subdomains $U_{n}=\operatorname{Sp} S_{n}$ such that on the subring $S_{n}^{0}$ of elements of bounded powers of $S_{n}$, there exists a finite-free module $M_{n}$ with a continuous action of $G$ such that $M_{n} \otimes_{S_{n}^{0}} S_{n}=V \otimes_{S} S_{n}$. By the proposition, we know that $H^{i}\left(G, M_{n}\right)$ is of finite type over $S_{n}^{0}$ and that $H^{i}\left(G, V \otimes S_{n}\right)=$ $H^{i}\left(G, M_{n} \otimes_{S_{n}^{0}} S_{n}\right)=H^{i}\left(G, M_{n} \otimes_{\mathbb{Z}_{p}} \mathbb{Q}_{p}\right)$ is of finite type over $S_{n_{0}} \otimes_{\mathbb{Z}_{p}} \mathbb{Q}_{p}=S_{n}$. But since $S_{n}$ is $S$-flat, $H^{i}\left(G, V \otimes_{S} S_{n}\right)=H^{i}(G, M) \otimes_{S} S_{n}$. The $S$-module $H^{i}(G, M)$ is thus finite over all open subdomains of an admissible covering. By Kiehl's Theorem ([BGR, Theorem 9.4.3/3]), it follows that $H^{i}(G, M)$ is finite over $S$. 


\subsection{Examples.}

2.4.1. The category $\mathcal{A}$. In all our examples, the $\operatorname{ring} R$ will be denoted $S$ and be either of the form $S_{0} \otimes_{\mathbb{Z}_{p}} \mathbb{Q}_{p}$, where $S_{0}$ is a Noetherian $p$-complete algebra, or a reduced affinoid algebra over $\mathbb{Q}_{p}$.

The category $\mathcal{A}$ is the category of finite $S$-modules, endowed with continuous $S$-linear action of $G_{K, \Sigma}$ (this makes sense since finite- $S$ modules have a canonical topology, the $p$-adic topology in the first case, and the natural topology (see e.g. [BGR, Prop 3.7.3/3]) in the second case).

Our functor $H_{\mathcal{A}}^{i}(-)$ from $\mathcal{A}$ to the category of $S$-modules is simply the continuous group cohomology $H^{i}\left(G_{K, \Sigma},-\right)$ defined by continuous cochains. Note that $\left(H^{i}\right)_{i \in \mathbb{N}}$ is a cohomological functor. Indeed, for any $0 \rightarrow A \rightarrow B \rightarrow C \rightarrow 0$, the map $B \rightarrow C$ admits a continuous set-theoretical section (even a continuous $\mathbb{Q}_{p}$-linear section: in the case where $S$ is affinoid over $\mathbb{Q}_{p}$, then $B$ and $C$ are complete $\mathbb{Q}_{p}$-vector spaces of countable type since so is $S$, and the existence of a continuous section results from [BGR, Prop. 7.2.1/4]; the other case is an exercise), so by standard results there is a long exact sequence of cohomology attached to this short exact sequence.

In particular, a family $V$ of representations of $G_{K, \Sigma}$ over $S$ is an object of $\mathcal{A}$. We fix such a family $V$. Obviously, since $V$ is locally free as an $S$-module it is torsion-free in $\mathcal{A}$ in the sense of $\$ 2.1$ and even quasi-free in $\mathcal{A}$ in the sense of $\$ 2.2$,

2.4.2. The $H_{T}^{1}$ 's. Let $S$ be a reduced affinoid algebra and let $T$ be a subset of $\Sigma_{p}$. We let $\mathcal{A}^{\prime}$ be the product of the categories $\mathcal{A}_{v}^{\prime}$ for $v$ in $T$, where $\mathcal{A}_{v}^{\prime}$ is the category of finite $S$-modules with a continuous action of $G_{v}$.

Let $H_{A^{\prime}}^{i}$ be the functor of continuous local Galois cohomology: $\left(A_{v}^{\prime}\right)_{v \in T} \rightarrow$ $\prod_{v \in T} H^{i}\left(G_{v}, A_{v}^{\prime}\right)$. For the same reason as for $\mathcal{A}$, the sequence $\left(H_{\mathcal{A}^{\prime}}^{i}\right)$ is a cohomological functor. Let $F: \mathcal{A} \rightarrow \mathcal{A}^{\prime}$ be the restriction functor, which obviously commutes with quotients.

We set $V^{\prime}=F(V)$. It is clear that $V^{\prime}$ is quasi-free in $\mathcal{A}^{\prime}$ in the sense of $\$ 2.2$, In this situation, the modules $S(V / I V)$ we get (for all ideals $I$ of $S$ ) are best noted as $H_{T}^{1}\left(G_{K, \Sigma}, V / I V\right)=\operatorname{ker}\left(H^{i}\left(G_{K, \Sigma}, V / I V\right) \rightarrow \prod_{v \in T} H^{i}\left(G_{v}, V / I V\right)\right)$. Two special cases are $T=\emptyset$, where we simply get the full Galois cohomology group $H^{1}\left(G_{K, \Sigma}, V / I V\right)$, and $T=\Sigma_{p}$.

Since the $H^{i}\left(G_{K, \Sigma}, V\right)$ and $H^{i}\left(G_{v}, V\right)$ are of finite-type (and 0 if $i>2$ ) by Corollary 3, we can apply Corollary 2, and we get in particular that there is an $r \geq 0$ such that for $x$ running among regular closed points of Spec $S$ such that $V_{x}^{G_{v}}=0$ for $v \in T$, the dimension of $H_{T}^{1}\left(G_{K, \Sigma}, V_{x}\right)$ is at least $r$ with equality for $x$ outside a Zariski closed proper subset.

Remark 3. This nice behavior is consistent with Jannsen's conjecture (see [J) in the cases where $S$ is an affinoid over $\mathbb{Q}_{p}$ and $T$ is either $\emptyset$ or $\Sigma_{p}$. Indeed, that conjecture says that the dimension of $H_{T}^{1}\left(G_{K, \Sigma}, W\right)$, for $W$ a geometric representation of motivic weight not equal to -1 over a finite extension of $\mathbb{Q}_{p}$, and $T$ either $\emptyset$ or $\Sigma_{p}$, only depends on simply computable local terms and global $H^{0}$, which are easily seen to be constant on a connected family.

In particular, looking for example at a refined family (see \$3.2.1) of representations $V$ of $G_{K, \Sigma}$ over $S$, there should be a dense set of points $x$ such that $V_{x}$ is geometric (either according to the Fontaine-Mazur conjecture or by construction of $V$ if $V$ is the family carried by an eigenvariety), and the condition of being of weight -1 defines a hypersurface of $S$, since the motivic weight can be written as 
a linear combination of the Hodge-Tate weights at places dividing $p$. Therefore, Jannsen's conjecture predicts that the dimension of $H_{T}^{1}\left(G_{K, \Sigma}, V_{x}\right)$ for $x$ geometric outside a hypersurface is constant, a prediction which is proved by the above result, and extended to non-geometric points.

In the intermediate cases where $T$ is not empty nor is $\Sigma_{p}$, we have heard of no conjecture predicting the dimension of $H_{T}^{1}\left(G_{K, \Sigma}, W\right)$. However, the above results suggest that such a conjecture, generalizing Jannsen's conjecture, should exist and have a simple form.

We plan to come back to these questions in a subsequent work.

2.4.3. The $H_{f}^{1}, H_{e}^{1}, H_{g}^{1}$ 's. Our second example is in fact a set of three counterexamples that we will not develop in full detail.

Let $\mathcal{A}^{\prime}$ be the product over $v$ in $\Sigma_{p}$ of categories $\mathcal{A}_{v}^{\prime}$, where $\mathcal{A}_{v}^{\prime}$ is a suitable abelian category of topological modules over $S$ with a continuous $G_{v}$-action. Let $H^{i}$ be the continuous cohomology functor as in the above example, and let $F_{f}: \mathcal{A} \rightarrow \mathcal{A}^{\prime}$ (resp. $\left.F_{e}, F_{g}\right)$ be the functor $V \rightarrow\left(V_{\mid G_{v}} \otimes B_{\text {crys }}\right)_{v \in \Sigma_{p}}$ (resp., with $B_{\text {crys }}$ replaced by $B_{\text {crys }}^{\phi=1}$, $\left.B_{\mathrm{dR}}\right)$, which obviously commutes with quotients.

In this case, we write $H_{f}^{1}\left(G_{K, \Sigma}, V / I V\right)$ (resp. $H_{e}^{1}$, resp. $\left.H_{g}^{1}\right)$ instead of $S(V / I V)$.

In those cases, the conclusion of Corollary 1 in general does not hold. The simplest way to see it is for $H_{e}^{1}$ for the family $\Lambda_{i, \mathbb{Q}_{p}}$ (with $i$ odd) described in the introduction. In this case, $K=\mathbb{Q}$, and $\Sigma=\Sigma_{p}$. As we have seen, for every $n \equiv i$ $(\bmod p-1)$, the representations $\mathbb{Q}_{p}(n)$ belong to that family. But for those representations we have $H_{e}^{1}\left(G_{\mathbb{Q}, \Sigma_{p}}, \mathbb{Q}_{p}(n)\right)=0$ if $n<0$ and $\operatorname{dim} H_{e}^{1}\left(G_{\mathbb{Q}, \Sigma_{p}}, \mathbb{Q}_{p}(n)\right)=1$ if $n$ is positive, contradicting the conclusion of Corollary 1, since obviously, we have $H^{0}\left(G_{p}, \mathbb{Q}_{p}(n) \otimes B_{\text {crys }}^{\phi=1}\right)=D_{\text {crys }}\left(\mathbb{Q}_{p}(n)\right)^{\phi=1}=0($ for $n \neq 0)$. One can infer that some hypotheses of the corollary do not hold. Since $H_{\mathcal{A}}^{i}\left(\Lambda_{i, \mathbb{Q}_{p}}\right)=H^{i}\left(G_{\mathbb{Q}, \Sigma_{p}}, \Lambda_{i} \otimes_{\mathbb{Z}_{p}} \mathbb{Q}_{p}\right)$ is finite for all $i$ by Proposition 3, we easily see that the hypothesis that is not satisfied here is the one that asks that $H^{1}\left(G_{v}, V \otimes B_{\text {crys }}^{\phi=1}\right)$ be of finite type over $\Lambda_{i, \mathbb{Q}_{p}}$.

2.4.4. Greenberg Selmer groups. Let $S$ be an affinoid algebra over $\mathbb{Q}_{p}$. In this example, we let $\mathcal{A}^{\prime}$ be the product of the categories $\mathcal{A}_{v}^{\prime}$ for $v$ in $\Sigma_{p}$, where $\mathcal{A}_{v}^{\prime}$ is the category of finite $S$-modules with a continuous action of $G_{v}$, and the functor $H_{\mathcal{A}^{\prime}}^{i}$ and $F$ are as in $\$ 2.4 .2$. But instead of taking $V^{\prime}=F(V)$, we start with a $V^{\prime}$ which is a quotient of $F(V)$. More precisely, we assume that $V^{\prime}=\left(V_{v}^{\prime}\right)_{v \in \Sigma_{p}}$, where each $V_{v}^{\prime}$ is a free $S$-module with a continuous action of $G_{v}$ which is a quotient of $V_{\mid G_{v}}$. If we call $b$ the canonical morphism $F(V) \rightarrow V^{\prime}$ in $\mathcal{A}^{\prime}$, we are exactly in the situation of 92.2 We can thus define for every ideal $I$ of $S$, Selmer groups $S(V / I V)$ that we can call the Greenberg Selmer Group $H_{\mathrm{gr}}^{1}\left(G_{K, \Sigma}(V / I V)\right)$ of $V / I V$ and $\left(V^{\prime} / I V^{\prime}\right)$ : we have $S(V / I V)=H_{\mathrm{gr}, V^{\prime}}^{1}\left(G_{K, \Sigma}, V\right)=\operatorname{ker}\left(H^{1}\left(G_{K, \Sigma}, V\right) \rightarrow \prod_{v \in \Sigma_{p}} H^{1}\left(G_{v}, V_{v}^{\prime}\right)\right)$.

The finiteness hypotheses of Proposition 2 are satisfied by Corollary 3 , Therefore, we see that for all points $x$ in $\operatorname{Sp} S$ outside a Zariski closed proper subset, the dimension of $H_{\mathrm{gr}, V^{\prime}}^{1}\left(G_{K, \Sigma}, V_{x}\right)$ is constant. A remarkable fact, due to Flach, is that for suitable choices of $V^{\prime}$ and $x$ (namely such that the Hodge-Tate weights of $\left(V_{v}^{\prime}\right)_{x}$ are exactly the Hodge-Tate weights of $\left.\left(V_{v}\right)_{x}\right)$ we have $H_{g}^{1}\left(G_{K, \Sigma}, V_{x}\right)=$ $H_{\mathrm{gr}, V^{\prime}}^{1}\left(G_{K, \Sigma}, V_{x}\right)$. This allows us to get some information about the behavior of the $H_{g}^{1}$ not given by the direct approach of $\$ 2.4 .3$ provided we can construct a suitable quotient $V^{\prime}$ of $F(V)$. Unfortunately, the conditions under which we can construct such a $V^{\prime}$, known as the Panchiskin condition, are too restrictive for the 
applications to eigenvarieties (except on their ordinary loci), and the aim of this paper is precisely to remove them.

\section{Trianguline families of $(\phi, \Gamma)$-MOdules}

\subsection{Families of $(\phi, \Gamma)$-modules and their triangulations.}

3.1.1. The relative Robba ring. Let $S$ be any ring with a non-archimedean complete valuation || . For any non-negative real $\rho<1$, we set

$$
\mathcal{R}_{S}^{\rho}=\left\{f=\sum_{i=-\infty}^{+\infty} a_{i} T^{i} \mid a_{i} \in S, f(T) \text { is convergent for } \rho \leq|T|<1\right\} .
$$

The convergence condition means that $\left|a_{i}\right| \rho^{i}$ goes to 0 when $i$ goes to $-\infty$ and that for every positive real number $r<1, a_{i} r^{i}$ goes to 0 when $i$ goes to $+\infty$. Therefore, for any real $r$ such that $\rho<r<1$, and any $f \in \mathcal{R}_{S}^{\rho}$, we can set $|f|^{(r)}=\sup _{i \in \mathbb{Z}}\left|a_{i} r^{i}\right|$.

For $f=\sum_{i \in \mathbb{Z}} a_{i} T^{i}$ and $g=\sum_{1 \in \mathbb{Z}} b_{i} T^{i}$ two elements of $\mathcal{R}_{S}^{\rho}$, then for every $i$ the sum $c_{i}=\sum_{j+k=i} a_{j} b_{k}$ converges and the series $\sum c_{i} T^{i}$ is an element of $\mathcal{R}_{S}^{\rho}$ that we call $f g$.

Lemma 1. The obvious addition and the multiplication just defined makes $\mathcal{R}_{S}^{\rho}$ a commutative ring, and the $\left.\right|^{(r)}$ are valuations on that ring. If $S$ is a Banach algebra over $\mathbb{Q}_{p}$, then those valuations make $\mathcal{R}_{S}^{\rho}$ a Frechet space over $\mathbb{Q}_{p}$ and a topological ring for its Frechet topology.

Note that for $1>\rho^{\prime}>\rho$, we have a continuous inclusion $\mathcal{R}_{S}^{\rho} \rightarrow \mathcal{R}_{S}^{\rho^{\prime}}$. We set

$$
\mathcal{R}_{S}=\bigcup_{\rho<1} \mathcal{R}_{S}^{\rho}
$$

The set $\mathcal{R}_{S}$ is obviously a ring (and an $S$ algebra), and it is called the (relative) Robba ring over $S$. When $S$ is a Banach algebra over $\mathbb{Q}_{p}, \mathcal{R}_{S}$ has a natural topology as an inductive limit of the Frechet space $\mathcal{R}_{S}^{\rho}$.

Lemma 2 (cf. Liu Liu2, Prop 3.2]). If $S$ is a reduced affinoid algebra over $K$ (with its spectral norm, which is a complete valuation), then $\mathcal{R}_{S}^{\rho}$ (resp. $\mathcal{R}_{S}$ ) is naturally isomorphic as a topological $S$-algebra to $\mathcal{R}_{\mathbb{Q}_{p}}^{\rho} \hat{\otimes} S$ (resp. to $\mathcal{R}_{\mathbb{Q}_{p}} \hat{\otimes} S$ ).

We shall also deal with the following subrings of $\mathcal{R}_{S}: \mathcal{E}_{S}^{\dagger}:=\left\{f=\sum a_{i} T^{i} \in\right.$ $\mathcal{R}_{S},\left(\left|a_{i}\right|\right)$ is bounded $\}, \mathcal{R}_{S}^{+}:=\left\{s=\sum_{i} a_{i} T^{i} \in \mathcal{R}_{S}, a_{i}=0\right.$ for $\left.i<0\right\}$, and $\mathcal{E}_{S}^{+}:=$ $\mathcal{E}_{S}^{\dagger} \cap \mathcal{R}_{S}^{+}$.

3.1.2. Different notions of families of $(\phi, \Gamma)$-modules. Let $S$ be an affinoid algebra over $\mathbb{Q}_{p}$.

By a general $(\phi, \Gamma)$-module over $S$ we mean a module $D$ over $\mathcal{R}_{S}$ with a semilinear action of $(\phi, \Gamma)$. Note the absence of conditions of finiteness or freeness of $D$, or of a topology on $D$. The category of general $(\phi, \Gamma)$-modules over $S$ is an abelian $S$-linear category (but not $\mathcal{R}_{S}$-linear).

By a family of $(\phi, \Gamma)$-modules (of rank $d$ ) over $S$, we mean a general $(\phi, \Gamma)$-module $D$ over $S$ such that $D$ is finite and projective over $\mathcal{R}_{S}$ (or, which amounts to the same, finite presentation and flat or finite presentation and locally free), such that for its natural topology (as a submodule of some free finite module over $\mathcal{R}_{S}$ ) the 
action of $\phi$ and $\Gamma$ are continuous, and such that $\phi(D)$ spans $D$ over $\mathcal{R}_{S}$. We say that a family $D$ of $(\phi, \Gamma)$-modules is free if $D$ is free over $\mathcal{R}_{S}$.

If $f: S \rightarrow S^{\prime}$ is a morphism of reduced affinoid algebras and $D$ is a general $(\phi, \Gamma)$-module over $S$, then $D_{S^{\prime}}:=D \otimes_{\mathcal{R}_{S}} \mathcal{R}_{S^{\prime}}$ has an obvious structure of general $(\phi, \Gamma)$-module over $S^{\prime}$. If $D$ is a family (resp. a free family), then so is $D_{S^{\prime}}$. We often adopt a geometric notation: if $X^{\prime}=\operatorname{Sp} S^{\prime}$ and $X=\operatorname{Sp} S$, we write $D_{X^{\prime}}$ instead of $D_{S^{\prime}}$ and we call it the pull-back of $D$ by the morphism $X^{\prime} \rightarrow X$ induced by $f$. We note that if $D$ is a free family of $(\phi, \Gamma)$-modules over $S$, then $D$ has a natural structure of Ind-Frechet vector space over $\mathbb{Q}_{p}$, so we can talk about $D \hat{\otimes}_{S} S^{\prime}$ and we have, using Lemma 2 ,

$$
D_{S^{\prime}}=D \hat{\otimes}_{S} S^{\prime}
$$

If $D$ is a family of $(\phi, \Gamma)$-modules over $S$ and $x \in \operatorname{Sp} S$, we denote by $D_{x}$ the $(\phi, \Gamma)$-module $D_{L(x)}$ over $\mathcal{R}_{L(x)}$ equal to $D \otimes_{\mathcal{R}_{S}} \mathcal{R}_{L(x)}=D \otimes_{S} \otimes L(x)$. It is a finite projective $\mathcal{R}_{L(x)}$-module. Since $\mathcal{R}_{L(x)}$ is an adequate Robba ring, every finite projective module is free (cf. e.g. [BCh, $\S 2.2]$ ). Thus $D_{x}$ is a finite free $\mathcal{R}_{L(x)^{-}}$ module.

3.1.3. The functor $D^{\dagger}$. Let $S$ be a reduced affinoid algebra. A construction of Berger and Colmez [BeCo] attaches to any family of representations $V$ of $G_{\mathbb{Q}_{p}}$ over $S$ a family of $(\phi, \Gamma)$-modules $D^{\dagger}(V)$, at least when $V$ admits a model $V_{0}$ over the algebra $S_{0}$ of power-bounded elements of $S$, that is, a finite locally free $S_{0}$-module with a Galois action such that $V_{0} \otimes_{S_{0}} S=V$. Chenevier has shown ([Ch, Lemma $3.18]$ ), using Raynaud's techniques that, at least locally (that is, on the constituents on an admissible affinoid covering of $\operatorname{Sp} S$ ), there always exists such a model $V_{0}$. This allows us to construct $D^{\dagger}(V)$ in general by gluing, and it is easy to prove that the result is independent of the choices made. Moreover, if $f: V \rightarrow V^{\prime}$ is a morphism of representations over $S$, then locally one can choose models $V_{0}$ and $V_{0}^{\prime}$ over $S_{0}$ and a morphism $f_{0}: V_{0} \rightarrow V_{0}^{\prime}$ such that $f_{0} \otimes S=f$. This results from the same Raynaud's techniques used by Chenevier. From that we deduce that $D^{\dagger}$ is a functor from the category of families of representations over $S$ to the category of families of $(\phi, \Gamma)$-modules over $S$ (the fact that it is a functor is stated without proof in $\mathrm{BeCo}]$.

Liu (cf. Liu2) has shown that this functor is fully faithful, and it is clear that it transforms exact sequences into exact sequences. Also, this functor commutes with arbitrary base change; cf. Ked.

Lemma 3. If $\pi: S p S^{\prime} \rightarrow S p S$ is a morphism of affinoid spaces and $V$ is a family of Galois representations over $S$, then one has a canonical isomorphism $D^{\dagger}\left(\pi^{*} V\right) \simeq D^{\dagger}(V) \hat{\otimes}_{S} S^{\prime}$

3.1.4. Free families of $(\phi, \Gamma)$-modules of rank one. Let $\delta: \mathbb{Q}_{p}^{*} \rightarrow S^{*}$ be a continuous character. We define a free family of $(\phi, \Gamma)$-modules $\mathcal{R}_{S}(\delta)$ of rank one as being $\mathcal{R}_{S}$ as a $\mathcal{R}_{S}$-module with semi-linear action of $\phi$ and $\Gamma$ given by $\phi(1):=\delta(p), \gamma(1):=$ $\delta(\gamma), \forall \gamma \in \Gamma$.

We shall say that the family of $(\phi, \Gamma)$-modules $\mathcal{R}_{S}(\delta)$ is of character type.

Lemma 4. Let $D$ be a free family of $(\phi, \Gamma)$-modules of rank one over $S$, and let $x \in S p S$. Then there exists an affinoid domain $S p S^{\prime} \subset S p S$ containing $x$ such that the restriction $D_{S^{\prime}}$ of $D$ to $S^{\prime}$ is isomorphic to $\mathcal{R}_{S^{\prime}}\left(\delta_{S^{\prime}}\right)$ for a unique continuous character $\delta_{S^{\prime}}: \mathbb{Q}_{p}^{*} \rightarrow S^{*}$. 
Proof. By twisting by a constant character we can reduce to the case where $D_{x}$ is etale. Then a theorem of Liu ([Liu3]) shows that there exists an affinoid domain $\operatorname{Sp} S^{\prime} \subset \operatorname{Sp} S$ containing $x$ such that the restriction $D_{S^{\prime}}$ of $D$ to $S^{\prime}$ is isomorphic to $D^{\dagger}$ of a continuous character of $G_{\mathbb{Q}_{p}}$ to $S^{\prime *}$. By local class field theory, this character defines a $\delta_{S^{\prime}}: \mathbb{Q}_{p}^{*} \rightarrow S^{\prime *}$, and it is clear that $D_{S^{\prime}}=\mathcal{R}_{S^{\prime}}\left(\delta_{S^{\prime}}\right)$.

Question 1. If $D$ is a free family of $(\phi, \Gamma)$-modules of rank one over $S$, is it globally of character type?

Of course, it is locally in a neighborhood of every point $x$, but there is a priori no reason that by using those neighborhoods one could make an admissible covering, which would allow one to glue the characters $\delta_{S^{\prime}}$.

3.1.5. Trianguline families. By a triangulation of a family $D$ of $(\phi, \Gamma)$-modules over $S$, we mean an increasing filtration $\left(\mathrm{Fil}^{i} D\right)_{i \in \mathbb{N}}$ of subfamilies such that $\mathrm{Fil}^{i} D$ is a family of rank $i$ for $i=0, \ldots, d$ and such that for all $i=1, \ldots, d, \operatorname{gr}^{i} D:=$ $\mathrm{Fil}^{i} D / \mathrm{Fil}^{i-1} D$ is of character type.

A family of $(\phi, \Gamma)$-modules provided with a triangulation (there may exist several) is called a trianguline family. A family of $(\phi, \Gamma)$-modules that admits a triangulation is called triangulable. Obviously, a triangulable family is free.

We say that a family of representations $V$ of $G_{\mathbb{Q}_{p}}$ over $S$ is trianguline if $D^{\dagger}(V)$ is, as a family of $(\phi, \Gamma)$-modules over $S$. If $\pi: \operatorname{Sp} S^{\prime} \rightarrow \operatorname{Sp} S$ is a morphism of affinoid and if $V$ is a family of representations over $S$, then the family $\pi^{*} V$ of representations of $G_{\mathbb{Q}_{p}}$ over $S^{\prime}$ is also trianguline, as it follows immediately from the definitions and Lemma 3 .

\subsection{Triangulation of some refined families.}

3.2.1. Definition of a refined family. We take the following definition from BCh, Chapter 4] except that instead of a pseudocharacter, we assume that our family is given by a free module with Galois action. The greater generality given by a pseudocharacter would be of no use here.

Definition 1. Let $X=\operatorname{Sp} S$ be a reduced affinoid space over $\mathbb{Q}_{p}$. A (rigid analytic) family of refined p-adic representations (or shortly, a refined family) of dimension $d$ over $X$ is a locally free module $V$ of dimension $d$ over $S$, together with a continuous $S$-linear action of $G_{\mathbb{Q}_{p}}$ and with the following data:

(a) $d$ analytic functions $\kappa_{1}, \ldots, \kappa_{d} \in \mathcal{O}(X)=S$,

(b) $d$ analytic functions $F_{1}, \ldots, F_{d} \in \mathcal{O}(X)=S$,

(c) a Zariski dense subset $Z$ of $X$;

subject to the following requirements:

(i) For every $x \in X$, the Hodge-Tate-Sen weights of $V_{x}$ are, with multiplicity, $\kappa_{1}(x), \ldots, \kappa_{d}(x)$.

(ii) If $z \in Z, V_{z}$ is crystalline (hence its weights $\kappa_{1}(z), \ldots, \kappa_{d}(z)$ are integers).

(iii) If $z \in Z$, then $\kappa_{1}(z)<\kappa_{2}(z)<\cdots<\kappa_{d}(z)$.

(iv) The eigenvalues of the crystalline Frobenius acting on $D_{\text {crys }}\left(V_{z}\right)$ are distinct and are $\left(p^{\kappa_{1}(z)} F_{1}(z), \ldots, p^{\kappa_{d}(z)} F_{d}(z)\right)$.

(v) For $C$ a non-negative integer, let $Z_{C}$ be the set of $z \in Z$ such that

$$
\kappa_{n+1}(z)-\kappa_{n}(z)>C\left(\kappa_{n}(z)-\kappa_{n-1}(z)\right) \text { for all } n=2, \ldots, d-1
$$


and $\kappa_{2}(z)-\kappa_{1}(z)>C$. Then for all $C, Z_{C}$ accumulates at any point of $Z$. In other words, for all $z \in Z$ and $C>0$, there is a basis of affinoid neighborhoods $U$ of $z$ such that $U \cap Z$ is Zariski-dense in $U$.

(*) For each $n$, there exists a continuous character $\mathbb{Z}_{p}^{*} \longrightarrow \mathcal{O}(X)^{*}$ whose derivative at 1 is the map $\kappa_{n}$ and whose evaluation at any point $z \in Z$ is the elevation to the $\kappa_{n}(z)^{\text {th }}$ power.

Recall that at every $z \in Z$, the representation $V_{z}$ is crystalline and has a natural refinement (that is, an ordering of its crystalline Frobenius eigenvalues), namely $\left(p^{\kappa_{1}}(z) F_{1}(z), \ldots, p^{\kappa_{d}}(z) F_{d}(z)\right)$. We call this refinement the canonical refinement of $V_{z}$.

We call $Z_{\text {ncr }}$ the set of $z \in Z$ such that the canonical refinement of $V_{z}$ is noncritical (see $[\mathrm{BCh}, \S 2.4 .3]$ ). That is, the filtration on $D_{\text {crys }}\left(V_{z}\right)$ given by the refinement is in generic position with respect to the Hodge-Tate filtration. By condition (v) and [BCh, Remark 2.4.6(ii)], one sees easily that the set $Z_{\text {ncr }}$ accumulates at any point of $Z$.

Also note that if $x \in Z$ and if $U \subset X$ is any affinoid subdomain of $X$, then the restriction of $V$ to $U$ (together with the restricted data $Z \cap U,\left(F_{i}\right)_{\mid U}$, etc.) is again a refined family over $U$. To make this true is indeed the main reason for the complicated form of condition (v), instead of simply asking if $Z_{C}$ is Zariski-dense. We shall use this remark freely in the sequel.

\subsubsection{Triangulation of families of rank 2 .}

Theorem 2. Let $(X, V)$ be a refined family of representations of $G_{\mathbb{Q}_{p}}$ of dimension 2. Let $x \in Z_{n c r}$ such that $V_{x}$ is irreducible and such that $F_{1}(x) / F_{2}(x) \notin p^{\mathbb{Z}}$. Then there is an affinoid subdomain $U$ of $X$ containing $x$ such that $D^{\dagger}\left(V_{U}\right)$ is trianguline, and the induced triangulation of $D^{\dagger}\left(V_{x}\right)$ is the one given by its canonical refinement.

Proof. For $i=1,2$, let $\delta_{i}: \mathbb{Q}_{p}^{*} \rightarrow S^{*}$ be defined by $\delta_{i}(p)=F_{i}$ and $\left(\delta_{i}\right)_{\Gamma}$ be the character $\mathbb{Z}_{p}^{*} \rightarrow S^{*}$ whose derivative at 1 is $-\kappa_{i}$, and evaluation at any $z \in Z$ is $t \mapsto t^{-\kappa_{i}(z)}$. The existence of such a character is property $(*)$ above, and its uniqueness is obvious.

Lemma 5. Up to replacing $X=$ Spec $S$ by a smaller affinoid containing $x$, there exist

(i) A triangular family $D$ of $(\phi, \Gamma)$-modules over $S$ such that for every $y \in S p S$, $D_{y}$ is a non-trivial extension of $\mathcal{R}_{L(y)}\left(\delta_{2, y}\right)$ by $\mathcal{R}_{L(y)}\left(\delta_{1, y}\right)$.

(ii) A free module $W$ of rank 2 over $S$ with a continuous action of $G_{\mathbb{Q}_{p}}$ such that $D^{\dagger}(W) \simeq D$.

Proof. Note that by weak admissibility and irreducibility of $V_{x}$, we have $\kappa_{1}(x)<$ $v_{p}\left(p^{\kappa_{i}(x)} F_{i}(x)\right)<\kappa_{2}(x)$ for $i=1,2$. It follows that $v_{p}\left(F_{2}(x)\right)<0<v_{p}\left(F_{1}(x)\right)$, so $v_{p}\left(\delta_{2, x}(p)\right)<v_{p}\left(\delta_{1, x}(p)\right)$. By continuity, after shrinking $X$ if necessary, we can assume that this property holds for all $y$ in $X$. We also have, for all $y \in X$, that $\kappa_{1}(y)+\kappa_{2}(y)=v_{p}\left(p^{\kappa_{1}(y)} F_{1}(y) p^{\kappa_{2}(y)} F_{2}(y)\right)$, so we have $v_{p}\left(\delta_{2, y}(p)\right)+v_{p}\left(\delta_{1, y}(p)\right)=0$.

Colmez calls $S S_{*}$ the rigid analytic moduli space of characters $\delta_{1}, \delta_{2}$ satisfying the condition $v_{p}\left(\delta_{2}(p)\right)<v_{p}\left(\delta_{1}(p)\right)$ and $v_{p}\left(\delta_{2}(p)\right)+v_{p}\left(\delta_{1}(p)\right)=0$. (See [Co1, $\S 0.2$ ], but note that he has weight conventions opposed to ours.) That is to say, the characters $\delta_{1}, \delta_{2}$ define an analytic map $\operatorname{Sp} S \rightarrow S S_{*}$, such that $\delta_{i}$ is the pull-back of the universal character $\tilde{\delta}_{i}$ for $i=1,2$. 
In [Co1, §5.1], Colmez constructs, locally on $S S_{*}$ near any point where $\tilde{\delta}_{1} \tilde{\delta}_{2}^{-1} \notin$ $p^{\mathbb{Z}}$, a family of $(\phi, \Gamma)$-modules of rank 2 whose fiber at every point $y$ is a non-trivial extension of $\tilde{\delta}_{2, y}$ by $\tilde{\delta}_{1, y}$. The pull-back $D$ to $S$ of this family of $(\phi, \Gamma)$-modules obviously satisfies (i).

To prove (ii), we note that by construction $D_{y}$ is an étale $(\phi, \Gamma)$-module. Therefore, by [Liu3, Main Theorem], there exists a representation $W$ on $S$ (up to shrinking it again) such that $D^{\text {rig }}(W)=D$.

To finish the proof of the theorem, it is enough to prove that $V \simeq W$ as $G_{\mathbb{Q}_{p}}$ representations over $X$, after shrinking $X$ if necessary.However,

Lemma 6. There exists an affinoid neighborhood $U$ of $x$ such that for every $z \in$ $Z_{n c r} \cap U$, we have $V_{z} \simeq W_{z}$ as $G_{\mathbb{Q}_{p}}$-representations.

Proof. By construction, $D^{\dagger}\left(W_{y}\right)$ is a non-trivial extension of $\mathcal{R}_{L(y)}\left(\delta_{2, y}\right)$ by $\mathcal{R}_{L(y)}\left(\delta_{1, y}\right)$ for every $y \in X$. By [Co1, Theorem 2.9], $\operatorname{Ext}^{1}\left(\mathcal{R}\left(\delta_{2, y}\right), \mathcal{R}\left(\delta_{1, y}\right)\right)$ has dimension 1 for $y$ in an affinoid neighborhood of $x$, and shrinking $X$ if necessary, for all $y$. Therefore, there exists up to isomorphism only one $(\phi, \Gamma)$-module that is a non-trivial extension of $\mathcal{R}_{L(y)}\left(\delta_{2, y}\right)$ by $\mathcal{R}_{L(y)}\left(\delta_{1, y}\right)$. Since $D^{\dagger}$ is an equivalence of category (over a base field), we only have to show that for $z \in Z_{\mathrm{ncr}}, D^{\dagger}\left(V_{z}\right)$ is such an extension.

By Kisin's theorem ([Kis, Corollary 5.16(c)], or [BCh, §3.3] in this context), we have

$$
D_{\text {crys }}\left(V_{z}\right)^{\phi=p^{\kappa_{1}(z)}} F_{1}(z) \neq 0 .
$$

Therefore, by [Co1, Prop 4.3], there exists an integer $k \in \mathbb{Z}$ such that if $\delta: \mathbb{Q}_{p}^{*} \rightarrow$ $L(z)^{*}$ is the character such that $\delta$ is $t \mapsto t^{k}$ on $\Gamma=\mathbb{Z}_{p}^{*}$ and $\delta(p)=p^{k+\kappa_{1}(z)} F_{1}(z)$, then $D^{\dagger}\left(V_{z}\right)$ has $\mathcal{R}(\delta)$ as a saturated sub- $(\phi, \Gamma)$-module. The integer $k$ is not explicitly determined in Co1, but actually it is easy to see that it is the opposite of the weight of the line $D_{\text {crys }}\left(V_{z}\right)^{\phi=p^{\kappa_{1}(z)}} F_{1}(z)$ (see [BCh, Prop 2.4.2]), that is, since $V_{z}$ is non-critical, $k=-\kappa_{1}(z)$. Thus $\delta=\delta_{1, z}$, and since $\mathcal{R}\left(\delta_{1, z}\right)$ is a saturated $(\phi, \Gamma)$-submodule of $D^{\dagger}\left(V_{z}\right)$, the quotient is a $(\phi, \Gamma)$-module of rank one which, by looking at the determinant, is seen to be $\mathcal{R}\left(\delta_{2, z}\right)$. Hence $D^{\dagger}\left(V_{z}\right)$ is an extension of $\mathcal{R}\left(\delta_{2, z}\right)$ by $\mathcal{R}\left(\delta_{1, z}\right)$, and this extension is non-trivial, since otherwise it would not be étale.

Going back to the proof of the theorem, we see using the lemma that $\operatorname{tr} V_{z}=\operatorname{tr} W_{z}$ for all $z \in Z_{\text {ncr }} \cap U$. Since (restricting $U$ again if necessary) $Z_{\text {ncr }} \cap U$ is Zariski-dense in $U$, we see that $\operatorname{tr} V=\operatorname{tr} W$ as maps $G_{\mathbb{Q}_{p}} \rightarrow S$. Since $\mathcal{O}_{X, y}$ (the rigid analytic local field of $X$ at $y$ ) is Henselian and since $V_{y}$ is irreducible, a famous theorem of Serre and Carayol (cf. Ca ) says that $V$ and $W$ are isomorphic over $\mathcal{O}_{X, y}$. Therefore, they are isomorphic over some affinoid neighborhood of $y$, which is what we wanted to prove.

Finally, the triangulation on $D_{x}$ that we have constructed is the unique one that has $\mathcal{R}\left(\delta_{1, x}\right)$ as $\mathrm{Fil}^{1} D_{x}$. Since the refinement of $V_{x}$ is non-critical, the attached triangulation of $D^{\dagger}\left(V_{x}\right)$ is the one whose $\mathrm{Fil}^{1}$ is also $\mathcal{R}\left(\delta_{1, x}\right)$ (cf. the proof of the above lemma). This proves the last assertion of the theorem.

Remark 4. If $V_{x}$ is reducible, a variant of this theorem has been known for a long time. Actually, if $V_{x}$ is reducible, then either $v_{p}\left(F_{1}(x)\right)$ or $v_{p}\left(F_{2}(x)\right)=0$. If $v_{p}\left(F_{1}(x)\right)=0$, we say that the point $x$, or the canonical refinement of $V_{x}$, is 
ordinary. In this case, the property $v_{p}\left(F_{1}\right)=0$ continues to be true on some neighborhood $U$ of $x$, and on $U$ the characters $\delta_{1}$ and $\delta_{2}$ are of Galois type (that is, $\delta_{i}(p)^{n}$ goes to 1 when $n$ goes multiplicatively to $\infty$-after shrinking $U$ again if necessary) and the family $V$ is well known to be an extension of the character $\delta_{2}$ by the character $\delta_{1}$. This obviously implies, and is much stronger than, the fact that $D^{\text {rig }}(V)$ on $U$ is trianguline.

Note also that in the case where $V_{x}$ is reducible and semi-simple, a refinement of $V_{x}$ is ordinary if and only if it is critical. If $V_{x}$ is not semi-simple, only one refinement is ordinary but both are critical.

\subsubsection{A conjecture about triangulation of refined families.}

Conjecture 1. Let $(X, V)$ be a refined family of representations of $G_{\mathbb{Q}_{p}}$ of any dimension d. Let $x \in Z_{n c r}$. Then there is an affinoid subdomain $U$ of $X$ containing $x$ such that $D^{\dagger}\left(V_{U}\right)$ is trianguline, and the induced triangulation of $D^{\dagger}\left(V_{x}\right)$ is the one given by the canonical refinement of $V_{x}$.

The case $d=1$ is trivial, and the results above show that this conjecture holds, except perhaps for some technical restrictions for $d=2$.

The infinitesimal variant of this conjecture (where we replace "there is an affinoid subdomain Sp $S^{\prime \prime}$ " by "for $S^{\prime}$ the completion of $S$ at $x$ ") is a theorem (again under a few technical restrictions): [BCh, Theorem 2.5.6]. The non-criticality assertion is critical in the proof (based on repeated application of Kisin's theorem on $\Lambda^{i} V$ for $i=1, \ldots, d)$ and is necessary for the result; see [BCh, Remark 2.5.8].

\subsection{Finiteness results for the cohomology of $(\phi, \Gamma)$-modules.}

3.3.1. Cohomology of a general $(\phi, \Gamma)$-module. Let $D$ be a general $(\phi, \Gamma)$-module over $\mathcal{R}$ (cf. 3.1.2).

As in Co1, §2.1], we define three $\mathbb{Q}_{p}$-vector spaces $H^{0}(D), H^{1}(D)$, and $H^{2}(D)$ as the cohomology spaces of the complex

$$
D \stackrel{d_{1}}{\rightarrow} D^{2} \stackrel{d_{2}}{\rightarrow} D
$$

with

$$
\begin{aligned}
d_{1}(c) & =((\gamma-1) c,(\phi-1) c) \\
d_{2}(a, b) & =(\phi-1) a-(\gamma-1) b .
\end{aligned}
$$

Of course, the construction of $H^{\bullet}(D)$ is functorial in $D$, so if $D$ has a structure of $S$-modules (where $S$ is a ring) compatible with the action of $\phi$ and $\Gamma$, then the spaces $H^{\bullet}(D)$ are naturally $S$-modules as well. Also, even in this generality, it is clear that any short exact sequence of $(\phi, \Gamma)$-modules $0 \rightarrow D_{1} \rightarrow D \rightarrow D_{2} \rightarrow 0$ gives rise to a long exact sequence

$$
0 \rightarrow H^{0}\left(D_{1}\right) \rightarrow \cdots \rightarrow H^{2}\left(D_{2}\right) \rightarrow 0
$$

In other words $\left(H^{i}\right)$ is a cohomological functor from the category of general $(\phi, \Gamma)$ modules over $\mathbb{Q}_{p}$ (resp. over $S$ ) to the category of $\mathbb{Q}_{p}$-vector spaces (resp. of $S$-modules). 


\subsubsection{Finiteness results.}

Proposition 4. Let $D$ be a free family of $(\phi, \Gamma)$-modules of rank one of character type over $S$. Then there exists an admissible covering of $S p S$ by affinoid subdomain $S_{\alpha}$, such that, upen replacing $S$ by any of the $S_{\alpha}$, the modules $H^{0}(D), H^{1}(D)$ and $H^{2}(D)$ are finite over $S$.

Proof. We have by hypothesis $D=\mathcal{R}_{S}(\delta)$, where $\delta: G_{\mathbb{Q}_{p}} \rightarrow S^{*}$ is a continuous character. Set $\alpha=\delta(p) \in S^{*}$. The open sets $\operatorname{Spec} S_{l, m}$ defined by the inequalities $l \leq v_{p}(\alpha(\alpha)) \leq m$ for $a, b \in \mathbb{Z}$ form an admissible affinoid covering of $S$. Restricting to one of those affinoid subdomains, we may and shall assume that $l \leq v_{p}(\alpha) \leq m$ on $\operatorname{Sp} S$ for some integers $l$ and $m$.

The proof we shall give is directly inspired by Colmez' computation of the $H^{0}$ and $H^{1}$ of a $(\phi, \Gamma)$-module of rank one over a field in [Co1, §2.2]. In his proof, Colmez uses several lemmas over the ring $\mathcal{R}_{L}$ and $\mathcal{E}_{L}^{\dagger}$ which, though they are stated only over a field $L$, work as well over a reduced affinoid algebra $S$ since their proofs amount to checking that some explicit series are convergent. This in turn amounts to computing the limits of the norm of their coefficients, a computation that takes exactly the same form in $L$ or in $S$ since in both cases this norm is multiplicative, that is, a valuation. This applies to Lemmas A.1, A.2, and A.4, as well as Corollary A.3 and Lemma 2.3.1 of [Co1. We shall use those results for $\mathcal{R}_{S}$ and $\mathcal{E}_{S}^{\dagger}$ without further comment below.

Cases of $H^{0}$. Since $H^{0}\left(\mathcal{R}_{S}(\delta)\right) \subset \mathcal{R}_{S}(\delta)^{\phi-1}$ and $S$ is Noetherian, the result will follow if we prove that $\mathcal{R}_{S}(\delta)^{\phi-1}=\mathcal{R}_{S}^{\alpha \phi-1}$ is finite over $S$.

Actually, we have $\mathcal{R}_{S}^{\alpha \phi-1}=\left(\mathcal{R}_{S}^{+}\right)^{\alpha \phi-1}$, where $\mathcal{R}_{S}^{+}=\left\{\sum_{i>0} a_{i} T^{i} \in \mathcal{R}_{S}\right\}$. Indeed, if $f=\sum_{i \in \mathbb{Z}} a_{i} T^{i} \in \mathcal{R}_{S}$ is not in $\mathcal{R}_{S}^{+}$, and $j$ is the largest negative integer $i$ such that $a_{i} \neq 0$, then one sees, using the fact that $\phi\left(T^{-1}\right)=T^{-p}+$ terms of lower degrees, that if $\phi(f)=\sum b_{i} T^{i}$, we have $b_{i}=0$ for all $i$ such that $p j<i<0$, and therefore one cannot have $\alpha \phi(f)=f$.

Now choose a non-negative integer $k$ which is strictly greater than $-v_{p}(\alpha)$. The operator $-\sum_{n=0}^{+\infty}(\alpha \phi)^{n}$ is a continuous inverse of $\alpha \phi-1$ on $T^{k} \mathcal{R}_{S}^{+}$by the proof of [C11, Lemma A1]. Therefore the $S$-linear map $\left(\mathcal{R}_{S}^{+}\right)^{\alpha \phi=1} \rightarrow \mathcal{R}_{S}^{+} / T^{k} \mathcal{R}_{S}^{+}$is injective, and since the latter has finite rank $k$ over $S$ which is Noetherian, the finiteness of $H^{0}$ follows.

Case of $H^{1}$, when $m<0$. We mimic the beginning of the proof of [Co1, Prop. 2.6]:

Let $(a, b) \in D^{2}$ such that $d_{2}(a, b)=0$ (see (6) ). According to [Co1, Cor A.3, Lemma A.4 and Lemma 2.3(i)], since $v_{p}(\alpha) \leq m<0$, there exists $c \in D$ such that if $\left(a_{1}, b_{1}\right)=(a, b)+d^{1}(c)$, then $a_{1} \in \mathcal{R}_{S}^{+}, b_{1} \in\left(\mathcal{E}_{S}^{\dagger}\right)^{\psi=0}$ and $(\delta(\gamma) \gamma-1) b_{1} \in\left(\mathcal{E}_{S}^{+}\right)^{\psi=0}$. In particular, in $D^{2}$ we have

$$
\operatorname{ker} d_{2}=\left(\mathcal{R}_{S}^{+} \times D^{\prime}\right)+\operatorname{Im} d^{1},
$$

where $D^{\prime}=\left\{b \in\left(\mathcal{E}_{S}^{\dagger}\right)^{\psi=0},(\delta(\gamma) \gamma-1) b \in\left(\mathcal{E}_{S}^{+}\right)^{\psi=0}\right\}$.

Let $k>-v_{p}(\alpha)$ be an integer. We claim that in $D^{2}$ we have the inclusion

$$
\left(T^{k} \mathcal{R}_{S}^{+} \times T^{k}\left(\mathcal{E}_{S}^{+}\right)^{\psi=0}\right) \cap \operatorname{ker} d_{2} \subset d_{1}\left(\mathcal{R}_{S}^{+}\right) .
$$

Indeed, if $(a, b) \in T^{k} \mathcal{R}_{S}^{+} \times T^{k}\left(\mathcal{E}^{+}\right)^{\psi=0}$ and $d_{2}(a, b)=0$, then by Co1, Lemma A.1], $(\delta(p) \phi-1)$ is an automorphism of $T^{k} \mathcal{R}_{S}^{+}$, so there exists $c \in \mathcal{R}_{S}^{+}$such that 
$(\delta(p) \phi-1) c=b$. Also, since $d_{2}(a, b)=0$, we have $(\delta(p) \phi-1)((\delta(\gamma) \gamma-1) c-a)=0$, so $(\delta(\gamma) \gamma-1) c=a$ and $d_{1}(c)=(a, b)$.

Using (7) and (8) we see that there is a surjective $S$-map

$$
\left(\mathcal{R}_{S}^{+} \times D^{\prime}\right) /\left(T^{k} \mathcal{R}_{S}^{+} \times T^{k}\left(\mathcal{E}_{S}^{+}\right)^{\psi=0}\right) \rightarrow \operatorname{ker} d_{2} / \operatorname{Im} d_{1}=H^{1}(D) .
$$

We are thus reduced to proving that $\mathcal{R}_{S}^{+} / T^{k} \mathcal{R}_{S}^{+}$is finite over $S$, which is clear, and that $D^{\prime} / T^{k}\left(\mathcal{E}_{S}^{+}\right)^{\psi=0}$ is finite. Since $\mathcal{E}_{S}^{+} / T^{k} \mathcal{E}_{S}^{+}$is finite, we only need to show that $D^{\prime} /\left(\mathcal{E}_{S}^{+}\right)^{\psi=0}$ is finite, that is, using the definition of $D^{\prime}$, that the endomorphism $\delta(\gamma) \gamma-1$ of $\left(\mathcal{E}_{S}^{+}\right)^{\psi=0}$ has finite cokernel. But this follows from [Co1, Lemma 2.3(ii)].

Case of $H^{1}$, general $v_{p}(\alpha)$. As in [Co1, $\left.\S 2.4\right]$, there is an $S$-linear operator $\partial$ : $H^{1}\left(\mathcal{R}_{S}\left(t^{-1} \delta\right)\right) \rightarrow H^{1}\left(\mathcal{R}_{S}(\delta)\right)$ where $t$ is the character $\mathbb{Q}_{p}^{*} \rightarrow S^{*}, t \mapsto t$. Mimicking Co1, $\mathrm{S} 2.4]$, it is easy to see that coker $\partial$ is finite over $S$. Therefore, $H^{1}\left(\mathcal{R}_{S}(\delta)\right)$ is finite if $H^{1}\left(\mathcal{R}_{S}\left(t^{-1} \delta\right)\right)$ is finite. By induction, $H^{1}\left(\mathcal{R}_{S}(\delta)\right)$ is finite if $H^{1}\left(\mathcal{R}_{S}\left(t^{-n} \delta\right)\right)$ is finite for some $n \in \mathbb{N}$. But since $v_{p}(\delta(p)) \geq m$ on $S$, taking $n \geq m+1$, we get that $H^{1}\left(\mathcal{R}_{S}\left(t^{-n} \delta\right)\right)$ by the preceding case, so $H^{1}\left(\mathcal{R}_{S}(\delta)\right)$ is finite.

Case of $H^{2}$. By Liu's duality theorem (cf. [Liu1]), the case of $H^{2}$ is reduced to the case of $H^{0}$.

Corollary 4. Let $D$ be a trianguline family of $(\phi, \Gamma)$-module over $S$. Then there exists an admissible covering of $\mathrm{SpS}$ by open affinoid subdomains $\mathrm{SpS}_{\alpha}$ such that upon replacing $S$ by $S_{\alpha}$, the modules $H^{0}(D), H^{1}(D)$ and $H^{2}(D)$ are finite over $S$.

Proof. This is obvious by the long exact sequence of cohomology and the preceding proposition.

3.4. The local Pottharst Selmer group. Let $S$ be a reduced affinoid algebra over $\mathbb{Q}_{p}, V$ a trianguline family of representation over $S$ of rank $d$, and $a$ an integer in $\{0, \ldots, d\}$. Following Pottharst $([\mathrm{P}])$, we define an $S$-linear application

$$
\begin{array}{r}
u: H^{1}\left(G_{\mathbb{Q}_{p}}, V\right)=\operatorname{Ext}_{G_{\mathbb{Q}_{p}}}^{1}(1, V) \stackrel{D^{\dagger}}{\rightarrow} \operatorname{Ext}_{(\phi, \Gamma) \text {-modules }}^{1}\left(1, D^{\dagger}(V)\right) \\
=H^{1}\left(D^{\dagger}(V)\right) \rightarrow H^{1}\left(D^{\dagger}(V) / \operatorname{Fil}^{a} D^{\dagger}(V)\right) .
\end{array}
$$

Above, 1 denotes both the trivial representation of dimension 1 over $S$ and the trivial $(\phi, \Gamma)$-module over $\mathcal{R}_{S}$. The morphism denoted $D^{\dagger}$ is the morphism induced by the functor $D^{\dagger}$ using the fact that $D^{\dagger}(1)=1$ and that $D^{\dagger}$ transforms exact sequences into exacts sequences, therefore extension, of 1 by $V$ into extensions of 1 by $D^{\dagger}(V)$, and the last map is the $H^{1}$ of the canonical surjection. We define $H_{\mathrm{pot}, a}^{1}\left(G_{\mathbb{Q}_{p}}, V\right)=\operatorname{ker}\left(H^{1}\left(G_{\mathbb{Q}_{p}}, V\right) \stackrel{u}{\rightarrow} H^{1}\left(D^{\dagger}(V) / \mathrm{Fil}^{a} D^{\dagger}(V)\right)\right)$.

The following result is a close variant of the main theorem of Pottharst's paper $[\mathrm{P}$, Theorem 3.1]. The hypotheses and the proof we give are different, relying only on results of $[\mathrm{BCh}$, Chapter II], and it is close in spirit to the proof of Flach's theorem relating Greenberg and Bloch-Kato Selmer groups.

Proposition 5. Assume that $S$ is a finite extension $L$ of $\mathbb{Q}_{p}$, that $V$ is a non-critical and non-exceptional trianguline representation over $L$, and that $a$ is the number of non-positive Hodge-Tate weights of $V$. Then $H_{p o t, a}^{1}\left(G_{\mathbb{Q}_{p}}, V\right)=H_{g}^{1}\left(G_{\mathbb{Q}_{p}, V}\right)=$ $H_{f}^{1}\left(G_{\mathbb{Q}_{p}}, V\right)=H_{e}^{1}\left(G_{\mathbb{Q}_{p}}, V\right)$.

Proof. We first show that $H_{g}^{1}=H_{f}^{1}=H_{e}^{1}$ for a trianguline non-exceptional representation. By [BK, Corollary 3.8.4] (for $H_{e}^{1}=H_{f}^{1}$ ) and [BK, Proposition 3.8] (for 
$\left.H_{g}^{1}=H_{e}^{1}\right)$, it is enough to show that 1 and $p$ are not eigenvalues of $\phi$ on $D_{\text {crys }}(V)$. Let $\left(\delta_{i}\right)_{i=1, \ldots, d}$ be the parameter of $V$ and let us note $\mathcal{D}_{\text {crys }}$ as the functor from the category of $(\phi, \Gamma)$-modules over $L$ to the category of filtered $\phi$-modules (such that $\left.D_{\text {crys }}=\mathcal{D}_{\text {crys }} \circ D^{\dagger}\right)$ constructed by Berger, namely $\mathcal{D}_{\text {crys }}(D)=D[1 / t]^{\Gamma}$. By an immediate dévissage, it is enough to show that 1 and $p$ are not eigenvalues of $\phi$ on $\mathcal{D}^{\text {crys }}\left(\delta_{i}\right)$ (a space of dimension 1 or 0 , in which case it has no $\phi$-eigenvalue at all). But we see at once that this space is not 0 if and only if $\delta_{i}(\gamma)=\gamma^{-n}$ for $\gamma \in \Gamma$, where $n$ is a rational integer, necessarily equal to the weight $s_{i}$ of the character $\delta_{i}$. In this case, the action of $\phi$ is $p^{n} \delta(p)$, so we see that the only case where this can be one or $p$ is when $\delta_{i}(t)=t^{n}$ or $\delta_{i}(t)=t^{n}|t|$ for all $t \in \mathbb{Q}_{p}^{*}$, that is, when $\delta_{i}$ is exceptional.

We now prove that $H_{\text {pot,a }}^{1}(V) \subset H_{g}^{1}\left(G_{\mathbb{Q}_{p}}, V\right)$ for a trianguline non-critical $V$. We have to show that an extension $U$ (in the category of $(\phi, \Gamma)$-modules) of 1 by $D^{\dagger}(V)$ whose push-forward as an extension of 1 by $D^{\dagger}(V) / \mathrm{Fil}^{a} D^{\dagger}(V)$ is trivial, is de Rham. But this amounts to showing that an extension of 1 by $\operatorname{Fil}^{a} D^{\dagger}(V)$ is de Rham (as a $(\phi, \Gamma)$-module). Such an extension is a trianguline $(\phi, \Gamma)$-module of rank $a+1$ which has for a parameter the sequence of characters $\delta_{1}, \ldots, \delta_{a}, 1$, whose weights are $s_{1}, \ldots, s_{a}, 0$. Since $V$ is non-critical and since $a$ is its number of non-positive Hodge-Tate weights, we have $s_{1}<s_{2}<\cdots<s_{a}<0$, and this $(\phi, \Gamma)$-module is de Rham by [BCh, Proposition 2.3.4].

Finally, we conclude the proof by showing that

$$
\operatorname{dim} H_{\mathrm{pot}, a}^{1}\left(G_{\mathbb{Q}_{p}}, V\right)=\operatorname{dim} H_{f}^{1}\left(G_{\mathbb{Q}_{p}}, V\right)
$$

for a trianguline, non-exceptional $V$. Indeed, since $D^{\dagger}$ is an equivalence of category (we are over a field $L$ ), it realizes an isomorphism $H^{1}\left(G_{\mathbb{Q}_{p}}, V\right) \simeq H^{1}\left(D^{\dagger}(V)\right.$ ) which identifies $H_{\mathrm{pot}, a}^{1}\left(G_{\mathbb{Q}_{p}}, V\right)$ with $\operatorname{ker}\left(H^{1}\left(D^{\dagger}(V)\right) \rightarrow H^{1}\left(D^{\dagger}(V) / F i l^{a} D^{\dagger}(V)\right)\right)$. Since

the characters $\delta_{i}$ in the parameter of $V$ are non-exceptional, we have $\operatorname{dim} H^{i}\left(\mathcal{R}_{L}\left(\delta_{i}\right)\right)$ $=0$ if $i=0$ or 2 and 1 if $i=1$. In particular, we get easily by dévissage (and descending induction on $i$, from $i=d$ to $i=0)$ that $H^{0}\left(D^{\dagger}(V) / \mathrm{Fil}^{i} D^{\dagger}(V)\right)=0$. Applying this for $i=a$ we get, using the long exact sequence attached to $0 \rightarrow$ $\mathrm{Fil}^{a} D^{\dagger}(V) \rightarrow D^{\dagger}(V) \rightarrow D^{\dagger}(V) / \mathrm{Fil}^{a} D^{\dagger}(V) \rightarrow 0$, that

$$
H_{\text {pot }, a}^{1}\left(G_{\mathbb{Q}_{p}}, V\right) \simeq H^{1}\left(\operatorname{Fil}^{a} D^{\dagger}(V)\right),
$$

and a new dévissage shows that this space has dimension $a$. On the other hand, since as we have seen $H^{0}\left(D^{\dagger}(V)\right)=0$, so that $H^{0}\left(G_{\mathbb{Q}_{p}}, V\right)=0$, the dimension of $H_{f}^{1}\left(G_{\mathbb{Q}_{p}}, V\right)$ is by [BK, Corollary 3.8.4] the number of non-positive Hodge-tate weights of $V$, that is, by hypothesis, $a$.

\section{Proof of Theorem 1}

Let $K$ be a number field, $p$ a prime number that is totally split in $K, \Sigma_{p}$ the set of places of $K$ above $p$, and $\Sigma$ a finite set of places containing $\Sigma_{p}$.

For any affinoid algebra $S$ over $\mathbb{Q}_{p}$, for any locally free module $V$ of rank $d$ over $S$ with a continuous action of $G_{K}$, such that $D_{v}:=D^{\dagger}\left(V_{\mid G_{v}}\right)$ is trianguline (that is, provided with a triangulation Fil $D_{v}$ ) and for any fixed sequences of integers 
$\underline{a}=\left(a_{v}\right)_{v \in \Sigma_{p}}, 0 \leq a_{v} \leq d$, we define the global Pottharst Selmer group as

$$
\begin{aligned}
H_{\mathrm{pot}, \underline{a}}^{1}\left(G_{K, \Sigma}, V\right) & =\operatorname{ker}\left(H^{1}\left(G_{K, \Sigma}, V\right)\right) \stackrel{u}{\rightarrow} \prod_{v \in \Sigma_{v}} H^{1}\left(G_{v}, D_{v} / \mathrm{Fil}^{a_{v}}\left(D_{v}\right)\right) \\
& =\left\{x \in H^{1}\left(G_{K, \Sigma}, V\right), \forall v \in \Sigma_{p}, x_{v} \in H_{\mathrm{pot}, a_{v}}^{1}\left(G_{v}, V\right)\right\} .
\end{aligned}
$$

In the first line, $u$ is the composition of restriction map

$$
H^{1}\left(G_{K, \Sigma}, V\right) \rightarrow \prod_{v \in \Sigma_{p}} H^{1}\left(G_{v}, V_{\mid G_{v}}\right)
$$

with the products of the maps $u_{v}: H^{1}\left(G_{v}, V_{\mid G_{v}}\right) \rightarrow H^{1}\left(D_{v} / F i l^{a_{v}} D_{v}\right)$ constructed (and denoted by $u$ ) in 3.4 This notion is taken from $[\mathrm{P}$, where it is called a triangulordinary Selmer group.

Over a field, we have the following interpretation:

Proposition 6. Assume that $S=L$ is a finite extension of $\mathbb{Q}_{p}$, and assume that for each $v \in \Sigma_{p}, V_{\mid G_{v}}$ is trianguline, non-critical, and non-exceptional, and that $a_{v}$ is the number of non-positive Hodge-Tate weights of $V_{\mid G_{v}}$ Then $H_{p o t, \underline{a}}^{1}\left(G_{K, \Sigma}, V\right)=$ $H_{g}^{1}\left(G_{K, \Sigma}, V\right)=H_{f}^{1}\left(G_{K, \Sigma}, V\right)=H_{e}^{1}\left(G_{K, \Sigma}, V\right)$.

Proof. This follows immediately from the definitions and Proposition 5.

We now prove the main theorem (Theorem 11). According to Corollary 4 , there exists a covering of SpS by open affinoid subdomains $S_{\alpha}$ such that upon replacing $S$ by $S_{\alpha}$, all the $S$-modules $H^{i}\left(D_{v} / \mathrm{Fil}^{a} D_{v}\right)$ are finite, for $i=0,1,2, a=0,1, \ldots, d$, and $v \in \Sigma_{p}$.

We are now in a position to apply the formalism developed in $\$ 2.2$ For our base ring $R$ we simply take $R=S$. Indeed, we can take for $\mathcal{A}$ the category of finite $S$-modules endowed with a continuous representation of $G_{K_{\Sigma}}$, and for $\left(H_{\mathcal{A}}^{i}\right)_{1 \in \mathbb{N}}$, we take the usual continuous cohomology groups, exactly as in 2.4.1. As for $\mathcal{A}^{\prime}$, we take the product, indexed by $\Sigma_{p}$, of copies the category of general $(\phi, \Gamma)$-modules (cf. 33.1 .2$)$, and for $H_{\mathcal{A}^{\prime}}^{i}$ we take the product over $\Sigma_{p}$ of the cohomology of $(\phi, \Gamma)$-modules (cf. \$3.3.1). We also need an $S$-linear functor $F$ from $\mathcal{A}$ to $\mathcal{A}^{\prime}$ which commutes with quotients: we simply take $F(V)=\prod_{v \in \Sigma_{p}} D_{v} / \mathrm{Fil}^{a_{v}} D_{v}$, and the commutation property is a special case of Lemma 3 . We need a morphism of functors $H_{\mathcal{A}}^{1} \rightarrow H_{\mathcal{A}^{\prime}}^{1} \circ F$, that is, for all $V$, a functorial morphism $H^{1}\left(G_{K, \Sigma}, V\right) \rightarrow \prod_{v \in \Sigma_{p}} H^{1}\left(D_{v} / \mathrm{Fil}^{a_{v}}\left(D_{v}\right)\right)$; we take the morphism $u$ defined above (see the lines following (10)). Finally, we take for $V$ our family, for $V^{\prime}$ we take $\prod_{v \in \Sigma_{p}} H^{1}\left(D_{v} / \mathrm{Fil}^{a_{v}}\left(D_{v}\right)\right)$, and for the morphism $b: F(V) \rightarrow V^{\prime}$ is the obvious one.

By definition, $S(V)=H_{\text {pot }, \underline{a}}^{1}\left(G_{K, \Sigma}, V\right)$, and for all closed points $x$ in $\operatorname{Sp} S$, corresponding to a prime ideal $\mathfrak{p}, S\left(V_{x}\right)=S(V / \mathfrak{p} V)=H_{\text {pot }, \underline{a}}^{1}\left(G_{K, \Sigma}, V / f V\right)$.

We want to apply Corollary 2 to this situation. We only have to check the hypotheses that $H^{i}\left(G_{K, S}, V\right), \prod_{v \in \Sigma_{v}} H^{i}\left(D_{v} / \mathrm{Fil}^{a_{v}} D_{v}\right)$ are finite over $S$. The first one follows from Proposition 3 and we already know the second one. We also need to check that $V$ and $V^{\prime}$ are quasi-free, but this is trivial.

Corollary 1 thus tells us that setting $r=r_{\left(a_{v}\right)}:=\operatorname{rk}_{S} S(V)$, there exists a $g \in S$, $g \neq 0$, such that for any regular point $x \in S p S$ satisfying $H^{0}\left(G_{K, \Sigma}, V_{x}\right)=0$, one has

with equality if $g(x) \neq 0$.

$$
\operatorname{dim} S\left(V_{x}\right) \geq r
$$


The same of course will hold if we restrict our attention to the subset of $x$ 's such that $V_{x}$ is, at each $v \in \Sigma_{K}$, non-critical, non-exceptional, and with exactly $a_{v}$ non-positive Hodge-Tate weights. But for those $x$, we know by Proposition 6 that $S\left(V_{x}\right)=H_{\mathrm{pot}, \underline{a}}^{1}\left(V_{x}\right)=H_{g}^{1}\left(G_{K, \Sigma}, V_{x}\right)=H_{f}^{1}\left(G_{K, \Sigma}, V_{x}\right)=H_{e}^{1}\left(G_{K, \Sigma}, V_{x}\right)$, so we get exactly the conclusion of Theorem 1, except that we are restricted to regular points. But the Hironaka's resolution of singularity is available for $S$, so we can find in particular a surjective morphism $\pi: S^{\prime} \rightarrow S$ with $S^{\prime}$ regular. Since $\pi^{*} V$ is trianguline at each place $v$ (see 33.1 .5 ), we can apply the result obtained above to $S^{\prime}$, from which we easily get the results for $S$.

\section{Application to modular forms}

In $\mathrm{BCh}$ the following result, a special case of the Bloch-Kato conjecture, is proved 3

Theorem 3 ([BCh, Cor. 8.1.4]). Let $f=\sum_{n \geq 1} a_{n} q^{n}$ be a newform of weight $k=2 k^{\prime} \geq 4$ and level $\Gamma_{0}(N)$, with $N$ prime to $p$. Let $\rho=\rho_{f}\left(k^{\prime}\right)$ be the Galois representation of $G_{\mathbb{Q}, \Sigma}$ attached to $f$ (where $\Sigma$ is the set of primes dividing $N p)$, normalized so as to have motivic weight -1 . Then if $\varepsilon(\rho, 0)=-1$ we have $\operatorname{dim} H_{f}^{1}\left(G_{\mathbb{Q}, \Sigma}, \rho\right) \geq 1$.

We want to extend this result:

Theorem 4. The theorem above also holds if $k=2$. In particular, if $E$ is an elliptic curve over $\mathbb{Q}$ with good reduction at $p$ and such that $\epsilon(E, 0)=-1$, then the p-adic Selmer group of $E$ has rank at least one.

Of course, the "in particular" follows from the first sentence by using the fact that for our elliptic curve $E$, there is a modular newform $f$ of weight 2 and level $\Gamma_{0}(N)$ such that $\rho_{f}(1)=V_{p}(E)$ and the fact that the Bloch-Kato Selmer group $H_{f}^{1}\left(\mathbb{Q}, V_{p}(E)\right)$ is the same as $\operatorname{Sel}_{p}(E) \otimes_{\mathbb{Z}_{p}} \mathbb{Q}_{p}$, where $\operatorname{Sel}_{p}(E)$ is the $p$-adic Selmer group of $E$.

Remark 5. (i) In the case where $p$ is an ordinary prime for $f$, there already exists two other proofs of this theorem (and of Theorem 4): one due to Nekovar and one due to Skinner and Urban.

(ii) In the case of an elliptic curve $E$, there are two other recent proofs of this result, by completely different methods. See [Kim and [D].

Proof. Let us prove Theorem 4. In view of Nekovar and Skinner-Urban's result, we may assume that $f$ is supersingular at $p$. The representation $\left(\rho_{f}(1)\right)_{\mid G_{p}}$, which is crystalline and irreducible, always admits two non-critical refinements $\mathcal{R}$ ([BCh, Remark 2.4.6(iii)]). Let us choose one of them, so we fix an ordering of the crystalline eigenvalues $\left(\phi_{1}, \phi_{2}\right)$. The $(\phi, \Gamma)$-module $D=D^{\dagger}\left(\left(\rho_{f}(1)\right)_{\mid G_{p}}\right)$ is thus trianguline, of parameter $\left(\delta_{1}, \delta_{2}\right)$ with $\delta_{1}(p)=p \phi_{i}, \delta_{2}(p)=\phi_{2}$ and $\delta_{1}(t)=t, \delta_{2}(t)=1$ for $t \in \Gamma=\mathbb{Z}_{p}^{*}$ (using the fact that the Hodge-Tate weights of $\rho_{f}(1)_{\mid G_{\mathbb{Q}_{p}}}$ are -1 and 0; see [BCh, Prop. 2.4.1]). By weak admissibility, and irreducibility, we have $-1<v_{p}\left(\phi_{i}\right)<0$ for $i=1,2$. Therefore, $\delta_{1}$ and $\delta_{2}$, and hence $\rho_{f}(1)_{\mid \mathbb{G}_{\mathbb{Q}_{p}}}$, are non-exceptional.

\footnotetext{
${ }^{3}$ Actually, it is proved under two assumptions, called $\operatorname{Rep}(4)$ and $\operatorname{AC}\left(\pi_{f, E}\right)$, which at that time were announced in the literature but a complete proof of which had not yet appeared. The situation is a little bit better now, as preprints of Morel, Shin, and a team led by Harris cover what it needs to give a proof of $\operatorname{Rep}(4)($ and $\operatorname{Rep}(n)$ for that matter); cf. $\underline{\mathrm{M}}]$, $\underline{\mathrm{Sh}}]$ and $\underline{\mathrm{H}}]$.
} 
Let $\mathcal{E}$ denote the eigencurve of tame level $N$. Then $(f, \mathcal{R})$ is a point $x$ of the $\mathcal{E}$. Let $X$ be an irreducible affinoid neighborhood of $x$ in an irreducible component of $\mathcal{E}$ through $x$. Then $X=\operatorname{Sp} S$, where $S$ is an affinoid domain of dimension 1 .

Since $\rho_{f}$ is irreducible, by Rouquier and Nyssen's theorem (cf. [Ro], $[\mathrm{Ny}]$ ), there exists (after replacing the base field $\mathbb{Q}_{p}$ by a finite extension $L$ if necessary, and shrinking $X)$ a Galois representation $V$ of $G_{\mathbb{Q}, \Sigma}(\Sigma$ being the set of rational primes dividing $N p$ ) of dimension 2 on $X$ whose trace is the restriction to $X$ of the canonical pseudocharacter carried by $\mathcal{E}$, normalized so that at a point $z \in X$ corresponding to a classical modular eigenform $g_{z}$ of weight $k_{z}=2 k_{z}^{\prime}$ and of level $\Gamma_{0}(N)$, we have $V_{z} \simeq \rho_{g_{z}}\left(k_{z}^{\prime}\right)$. We call $Z$ the set of such classical points. The family $V_{\mid G_{\mathbb{Q}_{p}}}$ over $X$ together with the set of classical points $Z$ is a refined family in the sense of 3.2 .1 This follows from the construction of the eigencurve; see [BCh, Prop. 7.5.13] for details. Note that for $z \in Z$ attached to a form $g_{z}$ of weight $2 k_{z}^{\prime}$, the Hodge-Tate weights of $V_{z}$ are $-k_{z}^{\prime}$ and $k_{z}^{\prime}-1$, so $V_{z}$ has exactly 1 non-positive weight.

By Theorem 2 (applicable since $\left(V_{x}\right)_{\mid G_{Q_{p}}}$ is irreducible, trianguline and noncritical, and its parameter satisfies $\left.\left(\delta_{2} \delta_{1}^{-1}\right)(p) \notin p^{\mathbb{Z}}\right)$, up to shrinking $\operatorname{Sp} S$ if necessary, the family $V_{\mid G_{p}}$ is trianguline (the triangulation of $\left(V_{x}\right)_{\mid G_{p}}=\left(\rho_{f}\right)_{\mid G_{p}}$ being associated with the chosen refinement). We now apply Theorem 1 for $\underline{a}=$ $\left(a_{v}\right)_{v \in \Sigma_{p}=\{p\}}=(1)$ : up to shrinking $\operatorname{Sp} S$ if necessary, we get that there exists an integer $r$ such that for all $x \in \operatorname{Sp} S$ such that $\left(V_{x}\right)_{\mid G_{p}}$ is non-critical, non-exceptional, and with exactly one non-negative Hodge-Tate weight, $\operatorname{dim} H^{1}\left(G, V_{x}\right) \geq r$ with equality up to a finite number of exceptions. We apply this to the set of points $Z^{\prime} \subset Z$ of $z$ that correspond to classical forms $g$ of weight $2 k^{\prime} \geq 4$ with a non-critical, non-exceptional refinement. It is easily seen using (v) of the definition of a refined family (3.2.1) that $Z^{\prime}$ is infinite. Therefore for an infinity of $z \in Z^{\prime}$, we have $\operatorname{dim} H_{f}^{1}\left(G_{\mathbb{Q}, \Sigma}, V_{z}\right)=r$. But as is well known, the sign $\varepsilon(0)$ is constant in a connected family, so by hypothesis, if a form $g$ of weight $2 k^{\prime}$ corresponds to a $z \in Z^{\prime}$, with $\rho_{g}\left(k^{\prime}\right) \simeq V_{z}$, we have $\varepsilon\left(\rho_{g}\left(k^{\prime}\right), 0\right)=-1$, and by Theorem 3 , $\operatorname{dim} H_{f}^{1}\left(G_{\mathbb{Q}, \Sigma}, \rho_{g}\left(k^{\prime}\right)\right) \geq 1$ for those $g$, so $r \geq 1$. It follows that $\operatorname{dim} H_{f}^{1}\left(G_{\mathbb{Q}, \Sigma}, \rho_{f}(1)\right) \geq 1$.

\section{ACKNOWLEDGMENT}

It is a pleasure to acknowledge the influences of the work of many people on this article. Greenberg's work on Selmer groups in Iwasawa type families, in particular his proof of the type finiteness of Galois cohomology group in those families, has been influential. As it would be clear to any reader from the number of references to his work, Colmez' emphasis on $(\phi, \Gamma)$-modules over the Robba ring, his wonderful idea of trianguline representations, and its results about them (and their generalizations, as in chapter 2 of my book with Chenevier, the recent work on Berger-Colmez, and various works of Ruochuan Liu) were also very important. If the author had to single out the most important influence on this work, it would be Pottharst's recent work on "triangulordinary" representations (that is, essentially, non-critical representations in the terminology of [BCh$)$, and especially its definition of the "triangulordinary Selmer group".

The author would like to thank Laurent Berger, Gaëtan Chenevier, Pierre Colmez, Brian Conrad, Matthew Emerton, Ralph Greenberg, Kiran Kedlaya, Ruochuan Liu, and Jay Pottharst for many useful conversations and emails. Also, this work was begun in May 2007 while the author was invited to the University of Padua, and 
the author would like to take this opportunity to thank Francesco Baldassarri and Bruno Chiarellotto for their kindness and their hospitality.

\section{REFERENCES}

[BeCo] L. Berger \& P. Colmez, Familles de représentations de de Rham et monodromie padique, in BeBrCo, pages 303-337. MR2493221 (2010g:11091)

[BeBrCo] L. Berger, C. Breuil, P. Colmez (editors), $p$-adic representations of $p$-adic groups I: Galois representations and $(\phi, \Gamma)$-modules, Astérisque 319 (2008)

[BCh] J. Bellaïche \& G. Chenevier, p-adic Families of Galois representations, Astérisque, 324, SMF (2009). MR2656025

[BK] Bloch \& Kato, Tamagawa Numbers of Motives in The Gorthendieck festschrift, vol. 1, Progress in Math 89, Birkhauser, 1990.

[BGR] W. Bosch, U. Güntzer \& R. Remmert, Non-Archimedean Analysis, Springer-Verlag, Berlin (1984). MR0746961 (86b:32031)

[Ca] H. Carayol, Formes modulaires et représentations galoisiennes à valeurs dans un anneau local complet, in $p$-adic monodromy and the Birch and Swinnerton-Dyer conjecture, Contemp. Math. 165, AMS (1994), 213-237. MR1279611(95i:11059)

[Ch] G. Chenevier, Une application des variétés de Hecke des groupes unitaires, to appear in $[\mathrm{H}$, available on http://www.math.polytechnique.fr/ chenevier/pub.html.

[Co1] P. Colmez, Représentations triangulines de dimension 2, in [BeBrCo, pages 213-258. MR.2493219 (2010f:11173)

[D] T. \& V. Dokchitser Root numbers and parity of ranks of elliptic curves, preprint, June 2009 (available on arxiv).

[E] Eisenbud, Commutative Algebra with a view toward algebraic geometry, GTM 150, Springer. MR 1322960 (97a:13001)

[G] R. Greenberg, On the structure of certain Galois cohomology group, Doc. Math. 2006, Extra Vol., 335-391. MR2290593 (2008b:11112)

[H] M. Harris et al., Stabilisation de la formule des traces, variétés de Shimura, et applications arithmétiques. Book project, available on http://www.institut.math. jussieu.fr/projets/fa/bp0.html.

[J] U. Jannsen, On the l-adic cohomology of varieties over number fields and its Galois cohomology, in Galois groups over $\mathbb{Q}$ (Berkeley, CA, 1987), 315-360, Math. Sci. Res. Inst. Publ., 16, Springer, New York, 1989. MR1012170 (90i:11064)

[Ked] K. Kedlaya, Slope filtration and $(\phi, \Gamma)$-modules in families, preprint, available on http://math.mit.edu/ ${ }^{\sim}$ kedlaya/papers/families.pdf

[KedLiu] K. Kedlaya and R. Liu, On families of ( $\phi, \Gamma)$-modules, arXiv:0812.0112v2 (2009).

[Kis] M. Kisin, Overconvergent modular forms and the Fontaine-Mazur conjecture, Invent. Math. 153(2) (2003), 373-454. MR1992017 (2004f:11053)

[Kim] B.D. Kim, The parity conjecture for elliptic curves at supersingular reduction primes, Compositio Math 143 (2007), 47-72. MR.2295194 (2007k:11091)

[La] M. Lazard, Les zéros des fonctions analytiques d'une variable sur un corps valué complet, Inst. Hautes Études Sci. Publ. Math., No. 14 (1962), 47-75. MR0152519 (27:2497)

[Liu1] R. Liu, Cohomology and Duality for $(\phi, \Gamma)-$ Modules over the Robba Ring, IMRN 2007. MR:2416996 (2009e:11222)

[Liu2] R. Liu, Slope Filtrations in Family, arxiv:0809.0331 (2008).

[Liu3] R. Liu, Families of $(\phi, \Gamma)$-modules, arxiv:0812.0112 (2008).

[Liu4] R. Liu, personal communication, emails to the author dated from June 2009 to July 2010.

[Liu5] R. Liu, On Families of Refined p-adic Representations, preprint in preparation, http://www.math.mcgill.ca/rliu/.

[M] S. Morel, On the cohomology of certain non-compact Shimura varieties, Annals of Mathematics Studies, 173, Princeton Univ. Press, Princeton, NJ, 2010. MR.2567740 (2011b:11073)

[N1] J. Nekovar, On the parity of ranks of Selmer groups II, Comptes Rendus de l'Acad. Sci. Paris, Serie I, 332 (2001), No. 2, 99-104. MR1813764 (2002e:11060)

[N2] J. Nekovar, Selmer complexes, S.M.F. Astérisque 310 (2006). MR.2333680(2009c:11176) 
[N3] J. Nekovar, On the parity of ranks of Selmer groups III, Doc. Math. 12 (2007), 243-274. MR 2350290 (2009k:11109)

[Ny] L. Nyssen, Pseudo-representations, Math. Annalen 306 (1996), 257-283. MR.1411348 (98a:20013)

[P] J. Pottharst, Triangulordinary Selmer Groups, arXiv:0805.2572 (2008) MR 2711680

[Ro] R. Rouquier, Caractérisation des caractères et pseudo-caractères, J. Algebra 180(2) (1996), 571-586. MR.1378546 (97a:20010)

$[\mathrm{Ru}] \quad$ K. Rubin, Euler Systems, Annals of Math. Studies 147, Princeton Univ. Press, N.J., 2000. MR 1749177 (2001g:11170)

[SkU] C. Skinner and E. Urban, Sur les déformations p-adiques de certaines représentations automorphes, Journal Inst. Math. Jussieu 5(4) (2006), 629-698. MR2261226 (2008a:11072)

[Sh] S. W Shin, Odd-dimensional Galois representations arising from some conpact Shiura varieties, preprint.

[T] John Tate, Relations between $K_{2}$ and Galois cohomology, Inventiones Math 36 (1976), 257-274. MR.0429837 (55:2847)

Department of Mathematics, MS 050, Brandeis University, 415 South Street, WalTham, MassachusetTs 02453

E-mail address: jbellaic@brandeis.edu 